\title{
Nrf2 prevents Notch-induced insulin resistance and tumorigenesis in mice
}

\author{
Dionysios V. Chartoumpekis, ${ }^{1}$ Yoko Yagishita, ${ }^{1}$ Marco Fazzari, ${ }^{1,2}$ Dushani L. Palliyaguru, ${ }^{1}$ \\ Uma N.M. Rao, ${ }^{3}$ Apostolos Zaravinos, ${ }^{4}$ Nicholas K.H. Khoo, ${ }^{1}$ Francisco J. Schopfer, ${ }^{1}$ Kurt R. Weiss, ${ }^{5}$ \\ George K. Michalopoulos, ${ }^{3}$ Ian Sipula, ${ }^{6}$ Robert M. O’Doherty, ${ }^{6}$ Thomas W. Kensler, ${ }^{1}$ \\ and Nobunao Wakabayashi ${ }^{1}$ \\ 'Department of Pharmacology and Chemical Biology, School of Medicine, University of Pittsburgh, Pittsburgh, \\ Pennsylvania, USA. ${ }^{2}$ Fondazione Ri.MED, Palermo, Italy. ${ }^{3}$ Department of Pathology, University of Pittsburgh, Pittsburgh, \\ Pennsylvania, USA. ${ }^{4}$ Department of Life Sciences, School of Sciences, European University Cyprus, Nicosia, Cyprus. \\ ${ }^{5}$ Department of Orthopaedic Surgery and ${ }^{6}$ Department of Medicine, Division of Endocrinology and Metabolism, University \\ of Pittsburgh, Pittsburgh, Pennsylvania, USA
}

Insulin resistance is associated with increased incidence and enhanced progression of cancers. However, little is known about strategies that can effectively ameliorate insulin resistance and consequently halt cancer progression. Herein, we propose that the transcription factor Nrf2 (also known as $\mathrm{Nfe2}$ /2) may be such a target, given its central role in disease prevention. To this end, we developed a mouse that overexpresses the Notch intracellular domain in adipocytes (AdNICD), leading to lipodystrophy-induced severe insulin resistance and subsequent development of sarcomas, as a model reflecting that Notch signaling is deregulated in cancers and shows positive associations with insulin resistance and fatty liver disease in humans. Nrf2 pathway activation was achieved by knocking down Keap1, a repressor of Nrf2, in the AdNICD background. Constitutively enhanced Nrf2 signaling in this setting led to prevention of hepatic steatosis, dyslipidemia, and insulin resistance by repressing hepatic lipogenic pathways and restoration of the hepatic fatty acid profile to control levels. This protective effect of Nrf2 against diabetes extended to significant reduction and delay in sarcoma incidence and latency. Our study highlights that the Nrf2 pathway, which has been induced by small molecules in clinical trials, is a potential therapeutic target against insulin resistance and subsequent risk of cancer.

Conflict of interest: The authors have declared that no conflict of interest exists.

Submitted: October 5, 2017 Accepted: January 30, 2018 Published: March 8, 2018

Reference information: JCI Insight. 2018;3(5):e97735. https:// doi.org/10.1172/jici.insight.97735

\section{Introduction}

Insulin resistance, often associated with obesity (1) in Westernized societies, is a major risk factor for type 2 diabetes that increases the risk for other comorbidities, including cardiovascular disease (2) and cancer (3, 4). The capacity of adipose tissue to secrete cytokines (5), such as leptin, and to buffer excess circulating lipids is important in the maintenance of insulin sensitivity. Several human genome loci affecting peripheral adipose storage capacity have been associated recently with insulin resistance (6). Interestingly, excessive amounts of adipose tissue in obesity and loss of adipose tissue in lipodystrophy syndromes both result in insulin resistance and fatty liver disease: the pathogenic mechanism they share is the disturbed capacity of adipose tissue (7). In obese subjects, the positive energy balance exceeds the capacity of adipose tissue to store the extra energy, resulting in increased levels of circulating free fatty acids and triglycerides, followed by ectopic lipid accumulation, in turn leading to dyslipidemia, hepatic steatosis, and, ultimately, insulin resistance and type 2 diabetes. In lipodystrophic individuals, the minute amounts or complete absence of adipose tissue leads to similar metabolic outcomes (8). The importance of adipose tissue storage capacity is evident in the presence of noninsulin-resistant obese and insulin-resistant nonobese subjects in clinical trials that can be attributed to better adipose storage capacity in the former and worse in the latter (9). Thus, lipodystrophic mice are relevant models of insulin resistance and related metabolic disturbances (hepatic steatosis) due to dysfunctional adipose tissue both in the context of obesity and lipodystrophy (10).

In this study, we employed a model of extreme insulin resistance resulting from partial lipodystrophy induced by inhibition of adipogenesis through enhanced Notch1 signaling in adipocytes; we used so called AdNICD mice, which overexpress Notch intracellular domain (NICD) and its target gene Hes1 at 
about 4-fold higher levels compared with control mice. This model that we developed previously (11) was selected not only because the metabolic phenotype (hyperglycemia, hypertriglyceridemia, fatty liver disease) it develops is relevant to insulin resistance and diabetes in humans, but also because Notch1 expression in the subcutaneous adipose tissue of HIV-positive patients treated with antiretrovirals is increased (12) (Supplemental Figure 1, A and B; supplemental material available online with this article; https:// doi.org/10.1172/jci.insight.97735DS1). Some of the antiretroviral-treated patients may eventually develop lipodystrophy (13), thus we could speculate a potential association of Notch1 overexpression and lipodystrophy development in these patients. Moreover, patients with premature aging disease due to $L M N A$ mutation (Hutchinson-Gilford progeria syndrome) show increased Notch signaling in their mesenchymal stem cells (14) that leads to almost complete loss of their potential to differentiate to adipocytes. These patients usually show progressive and generalized fat loss, especially in their subcutaneous areas, and develop an insulin-resistant phenotype $(15,16)$, as also seen in mice that carry a mutant form of Lmna $(17)$, though their insulin resistance is milder than in other types of lipodystrophy. Another interesting feature of these AdNICD mice, as we describe in this work, is the development of tumors (sarcomas) when they age. Notch1 has been described to show aberrant expression in a variety of human cancers, including sarcomas (18-20), making this mouse attractive from a clinical perspective as an investigational model to study in parallel methods to prevent insulin resistance and cancer development.

Nuclear factor, erythroid derived 2, like 2 (Nrf2) is a ubiquitously expressed transcription factor that regulates a series of prosurvival genes (21), with an emerging protective role in type 2 diabetes in mice (22) and humans (23). The Nrf2 pathway has been targeted in our previous cancer chemoprevention clinical trials with small-molecule activators (24). Accrued evidence indicates that the beneficial effects of Nrf2 cannot be solely ascribed to its direct transcriptional effects but also to its crosstalk with other pathways $(25$, 26). Hence, we asked whether Nrf2 can exert a protective effect against both insulin resistance and cancer in our AdNICD model. To this end, the Nrf2 pathway was activated by introducing kelch-like ECH-associated protein 1 (Keap1) homozygous knockdown alleles into AdNICD mice (Keap1KD:AdNICD mice). Keap1KD mice are an established genetic model of Nrf2 pathway activation, as Keap1 sequesters Nrf2 in the cytoplasm and facilitates its proteasomal degradation (27). Hence, these Keap1KD mice express lower levels of Keap1 (28) and have constitutively enhanced systemic Nrf2 signaling without showing developmental defects, as seen in Keap1-null mice that die before weaning due to malnutrition caused by esophageal stricture (29).

We report here that Nrf2 pathway activation by knocking down Keap1 can prevent the development of severe insulin resistance in 3-month-old AdNICD mice by completely protecting them from the development of fatty liver disease and dyslipidemia through repression of hepatic lipogenic pathways. This protective effect was not due to rescue of the loss of adipose tissue. Nonetheless, it was so profound that the metabolic phenotype of Keap1KD:AdNICD mice was similar to that of control mice. The Keap1KD:AdNICD metabolic phenotype was also similar to the phenotype resulting from lipodystrophy rescue by adipocyte-specific deletion of Rbpjk (recombination signal binding protein for immunoglobulin $\kappa \mathrm{J}$ region) in AdNICD mice. Rbpjк is a core DNA-binding factor of NICD and a major component of Notch canonical pathway. Deletion of Nrf2 in the Keap1KD:AdNICD mice abrogated the protective effect afforded by Keap1KD, showing that the observed protection is totally Nrf2 dependent. Furthermore, the Keap1KD:AdNICD mice developed sarcomas at a significantly lower incidence and at median age of approximately 14.5 months, much later than the AdNICD mice (median age 7.5 months). Thus, enhanced Nrf2 signaling can exert a profound protective effect against insulin resistance and progression of tumorigenesis.

\section{Results}

Nrf2 pathway activation prevents metabolic dysregulation and insulin resistance in lipodystrophic mice. The compound AdNICD mice with submaximal activation of the Nrf2 pathway (Keap1KD:AdNICD) weighed less than AdNICD mice, similar to control mice, at 3 months of age (Figure 1A). Deletion of Nrf2 in the Keap1KD:AdNICD mice (Nrf2KO:Keap1KD:AdNICD mice) tended to restore body weight back to the higher AdNICD levels (Figure 1A). Keap1KD:AdNICD mice, however, were not rescued from lipodystrophy per se, as they almost completely lack white adipose tissue, like AdNICD mice, as is evident by macroscopic observation and by NMR-calculated total fat mass of these mice (Figure 1B). Similarly, food consumption was not affected in AdNICD mice by Nrf2 pathway activation (Figure 1C); however, both AdNICD and Keap1KD:AdNICD mice tended to consume larger amount of food per body weight and larger absolute 
A

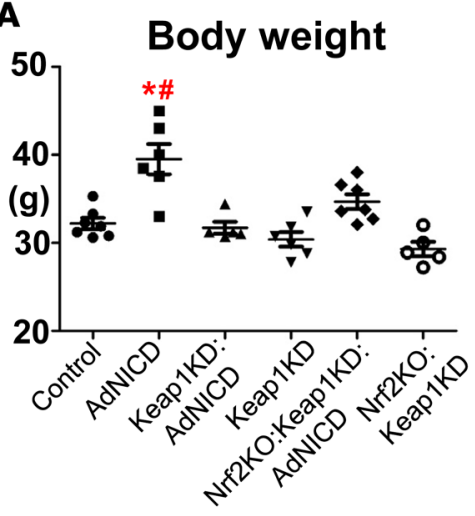

D

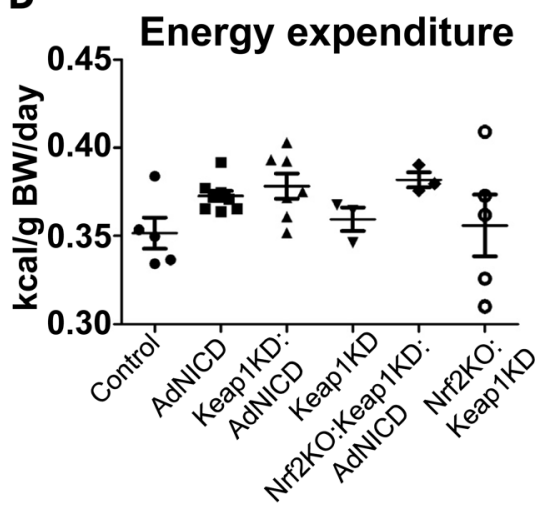

$\mathbf{F}$

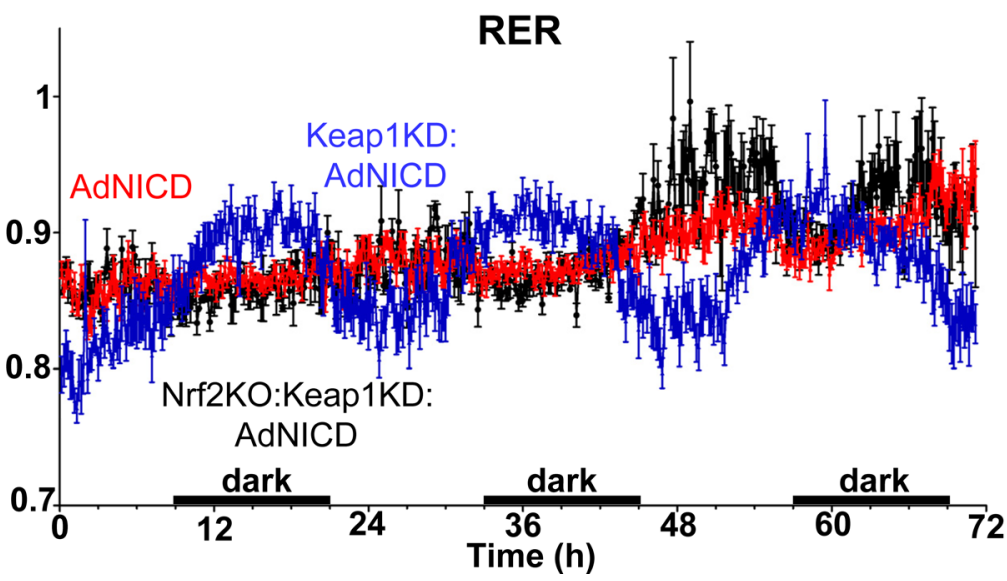

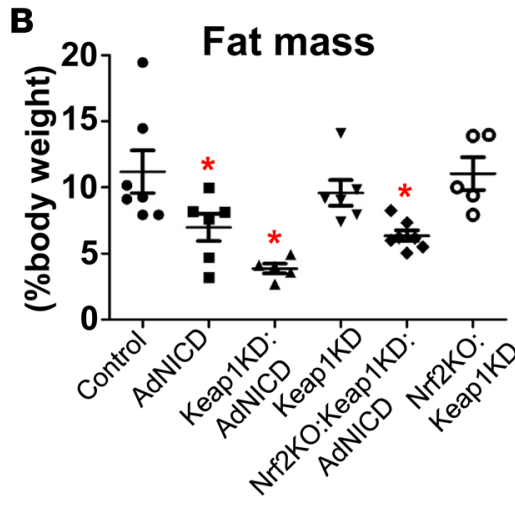

C
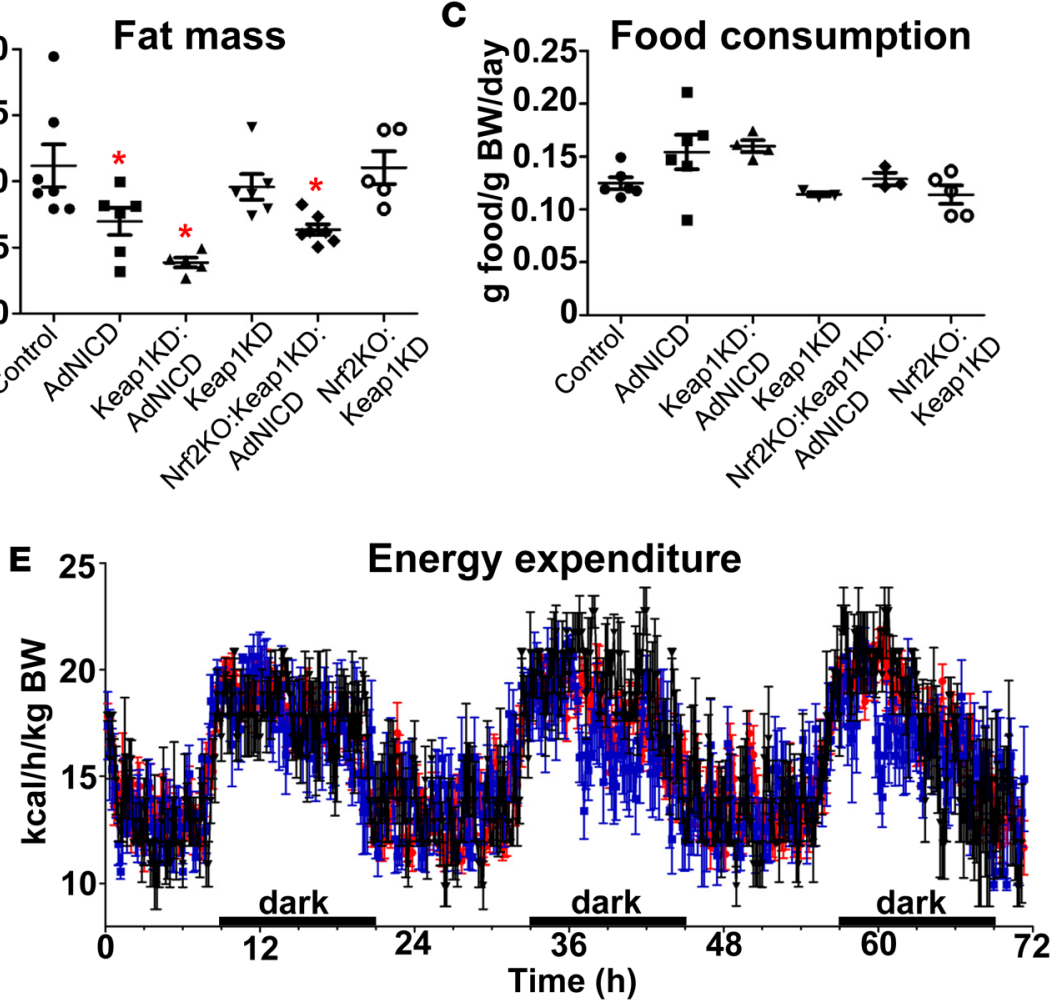

G

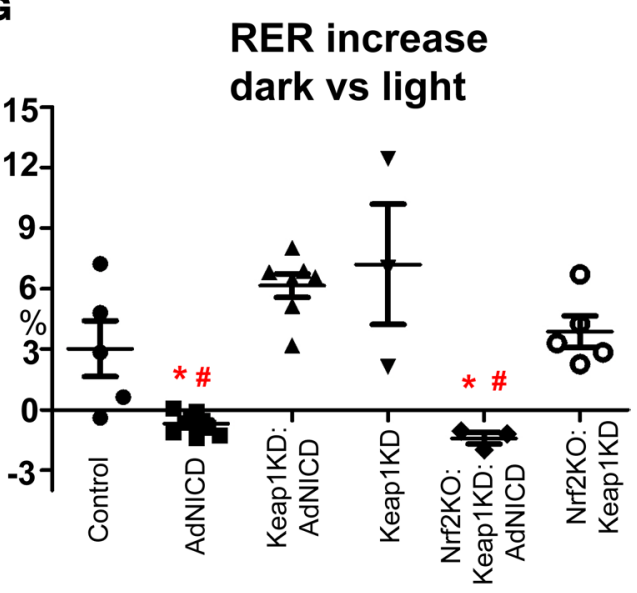

Figure 1. Activation of the Nrf2 pathway prevents energy metabolism disturbance in AdNICD lipodystrophic mice. (A) Body weights, (B) quantitative magnetic resonance-based measurement of fat mass, and (C) food consumption assessment over a 3-day period in 3-month-old male mice, plotted as body weight-corrected food consumption per day. (D) Energy expenditure assessment over a 3-day period in 2.5-month-old male mice plotted as body weight-corrected heat production per day. (E) Heat production and (F) respiratory exchange ratio (RER) tracing of mice of 3 genotypes (red line: AdNICD; blue line: Keap1KD:AdNICD; black line: Nrf2KO:Keap1KD:AdNICD with $n=9, n=7$, and $n=3$, respectively) over a period of 72 hours. (G) Percentage increase in RER in dark vs. light cycle (based on indirect calorimetry data from 72 hours). All data represent mean \pm SEM. ${ }^{*} P<0.05$ compared with control mice; ${ }^{\#} P<0.05$ compared with Keap1KD:AdNICD mice, 1-way ANOVA.

amounts compared with controls (Figure 1C and Supplemental Figure 1C). Enhanced Nrf2 pathway activation did not affect energy expenditure in the AdNICD background (Figure 1D), but AdNICD mice tended to show higher energy expenditure compared with controls (Figure 1D and Supplemental Figure 1, D-F). Interestingly, although energy expenditure of the AdNICD mice followed the expected light-dark oscillations (Figure 1E), as did their activity (Supplemental Figure 2A), their respiratory exchange ratio (RER = $\mathrm{VCO}_{2} / \mathrm{VO}_{2}$ ) oscillation was disturbed (Figure $1 \mathrm{~F}$ and Supplemental Figure $2 \mathrm{~B}$ ). Given that mice were fed a diet that provides calories mostly from carbohydrates, it was expected that the RER of normal mice should 
A

Blood glucose

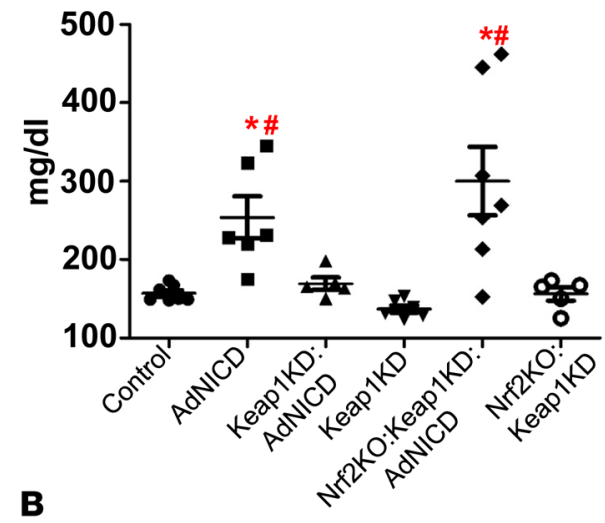

B

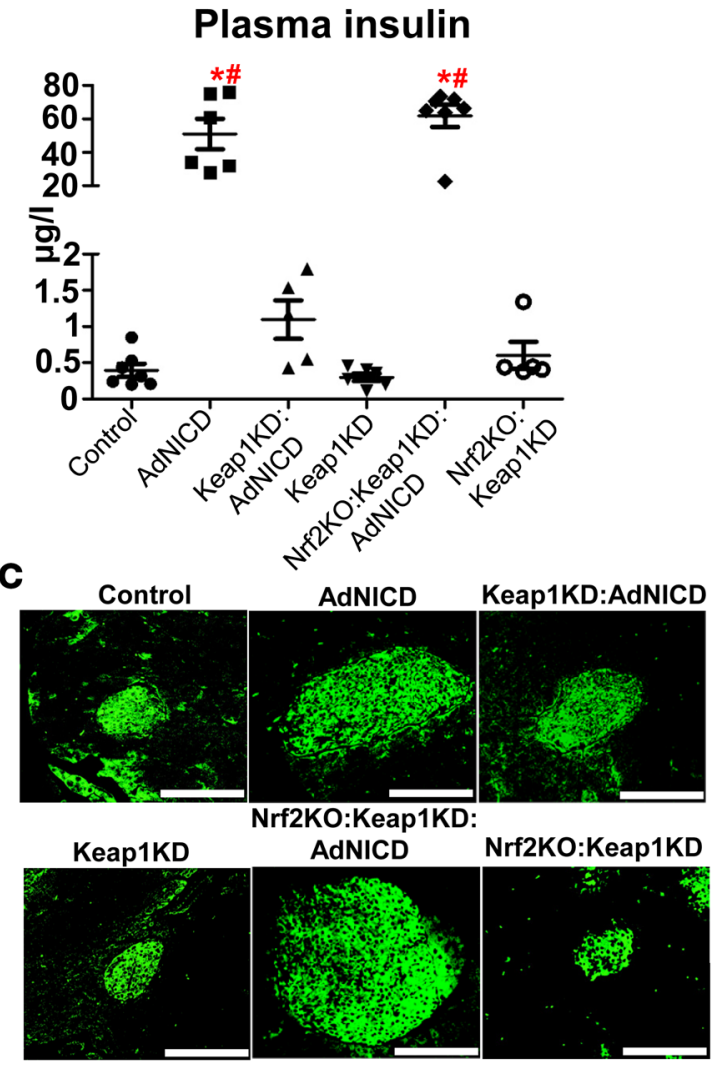

\section{Insulin positive area per islet}

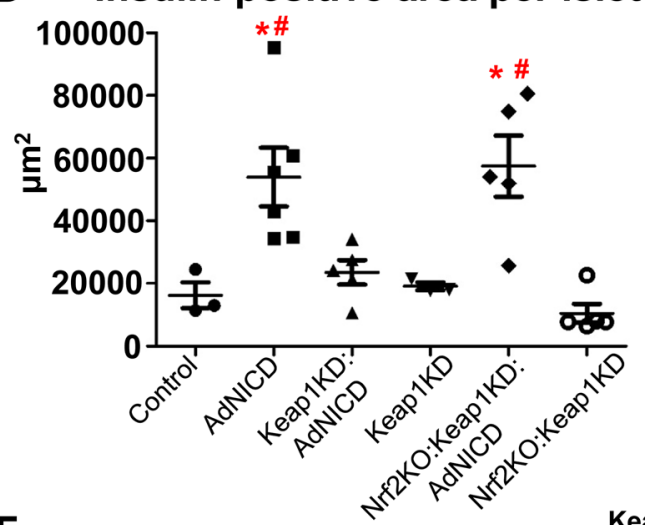

E

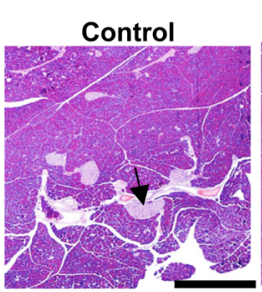

AdNICD
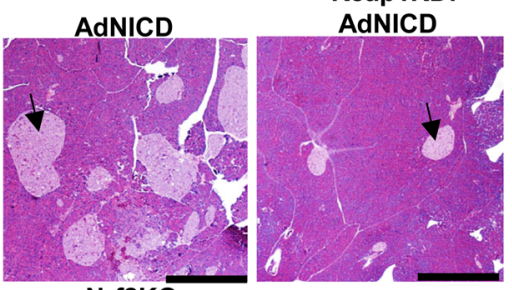

Keap1KD
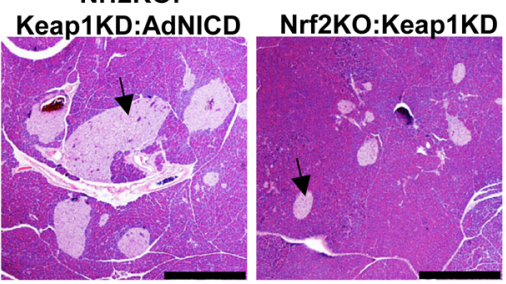

IPIST

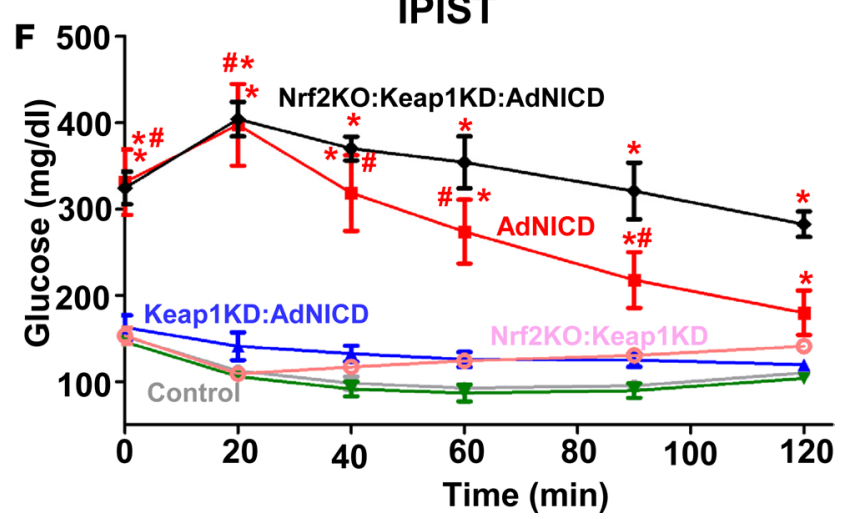

Figure 2. Nrf2 pathway activation protects AdNICD mice from insulin resistance. (A) Fed blood glucose levels and (B) plasma insulin levels. (C) Representative images of immunofluorescent staining of insulin (green) in 3-month-old mice (scale bar: $50 \mu \mathrm{m}$ ) and (D) quantification of the insulin-positive area per islet. (E) Representative images of H\&E-stained sections of pancreata of 3-month-old male mice. Arrowheads point to islets. Scale bar: $500 \mu \mathrm{m}$. (F) Blood glucose levels during an intraperitoneal insulin sensitivity test (IPIST) in 2-month-old mice of all genotypes. Control ( $n=7)$, AdNICD ( $n=4)$, Keap1KD:AdNICD $(n=5)$, Keap1KD $(n=6)$, Nrf2KO:Keap1KD:AdNICD $(n=5)$, Nrf2KO:Keap1KD $(n=5)$. All data represent mean \pm SEM. ${ }^{*} P<0.05$ compared with control mice; $\# P$ $<0.05$ compared with Keap1KD:AdNICD mice, 1-way ANOVA (A, B, and D) and 2-way ANOVA (F).

reach a peak during the dark cycle. Conversely, during the light cycle, mice relied mostly on oxidation of fatty acids for energy production, which resulted in lower RER (Supplemental Figure 2C). As is depicted in Figure 1F, activation of Nrf2 in Keap1KD:AdNICD mice restored the RER oscillation to a regular lightdark cycle similar to that of controls, whereas deletion of Nrf2 in the Keap1KD:AdNICD mice led to a similarly disturbed RER as in AdNICD mice. Hence, AdNICD mice did not show the expected increase of RER in the dark cycle, while Keap1KD:AdNICD mice showed a RER increase similar to that in controls; Nrf2 deletion led to loss of RER increase, indicating that this effect is Nrf2 dependent (Figure 1G and Supplemental Figure 2, D and E). 
This observed disturbance in RER oscillation can be indicative of severe insulin resistance that impairs tissue uptake of glucose and its use for energy production. Indeed, also indicative of extreme insulin resistance, AdNICD mice had significantly higher blood glucose (1.6-fold) (Figure 2A) and plasma insulin (128-fold) (Figure 2B) levels compared with control mice at 3 months of age. Genetic activation of the Nrf2 pathway in Keap1KD:AdNICD mice resulted in complete prevention of hyperglycemia and hyperinsulinemia, while Nrf2 deletion in Nrf2KO:Keap1KD:AdNICD mice abrogated this protective effect (Figure 2, A and B). The insulin-positive area in the pancreatic islets of Langerhans was also markedly increased (3.3-fold) in AdNICD mice to compensate for the extreme peripheral insulin resistance; however, the insulin-positive area in islets of Keap1KD:AdNicD mice was similar to that of control animals, yet another indication that activation of Nrf2 signaling prevented insulin resistance (Figure 2, C and D). These changes are also roughly reflected in the size of the pancreatic islets (Figure 2E).

An intraperitoneal insulin sensitivity test further substantiated the profound preventive effect of Nrf2 against insulin resistance (Figure $2 \mathrm{~F}$ and Supplemental Figure 2F), as Keap1KD:AdNICD mice showed fast clearance of glucose from their blood upon insulin administration, similar to control mice, while AdNICD mice were able to reduce their circulating glucose levels only after 60 minutes following insulin injection. Lack of Nrf2 in the Keap1KD:AdNICD background resulted in a similar response to insulin as in AdNICD mice, further establishing that the protection against insulin resistance by Keap1KD is Nrf2 dependent.

Nrf2 protects AdNICD mice from hypertriglyceridemia and fatty liver disease. Circulating triglycerides (Figure 3A) and free fatty acids (Figure 3B) returned from approximately 5-fold and approximately 1.5 -fold levels, respectively, in the AdNICD lipodystrophic mice back to control levels in the 3-month-old Keap1KD:AdNICD mice in a Nrf2-dependent manner. Modest changes were seen in cholesterol in AdNICD mice only (Figure 3C). The plasma levels of leptin, a hormone that is secreted mainly by adipocytes, were very low in the lipodystrophic mice, as expected, and were not affected by Nrf2 pathway activation status (Figure 3D). This gives further support to our observation that Nrf2 does not change the fate of adipose tissue in AdNICD mice and, thus, lipodystrophy itself is not rescued. Nevertheless, activated Nrf2 signaling completely prevented the development of macrovesicular and microvesicular hepatic steatosis, as evidenced by pathologic examination of $\mathrm{H} \& \mathrm{E}$-stained liver sections (Figure $3 \mathrm{E}$ ). While the absolute protection against steatosis by Nrf2 was not reflected in any changes in the profile of hepatic triglycerides species as compared with Keap1KD:AdNICD mice (Figure 4A), the total amount of hepatic triglycerides, which rose 12.7-fold in AdNICD mice, returned to control levels with Nrf2 activation (Figure 4B). This protection was abrogated when Nrf2 was deleted in Nrf2KO:Keap1KD:AdNICD mice (Figure 4B).

To evaluate the contribution of another major tissue to the resulting phenotype, muscle triglycerides were assessed. The muscle triglyceride profile revealed not only that activation of Nrf2 signaling did not affect the overall distribution of various triglycerides species, but that muscle did not accumulate excess triglycerides in AdNICD mice, as happens in the liver (Figure 4C). Quantification of the total levels of triglycerides clearly showed that AdNICD mice had low levels of triglycerides in their muscles and that Nrf2 did not affect these levels (Figure 4D). This means that the protective role of Nrf2 against diabetes in AdNICD mice is largely mediated by the liver rather than the muscle. The profound effect of Nrf2 on the liver was also shown by the extent of protection that Nrf2 confers against hepatomegaly in AdNICD mice (Figure 4E), which should be mainly attributed to prevention of hepatic triglyceride accumulation by Nrf2. These data led us to focus our study on the liver to investigate how Nrf2 profoundly prevents fatty liver disease in the AdNICD mice.

Nrf2 represses lipogenic enzymes in the livers of AdNICD lipodystrophic mice. The transcript levels of major de novo lipogenesis enzymes Fasn (Figure 5A) and Acaca (Figure 5B) were induced approximately 2- fold in 3-month-old AdNICD mice. Activation of the Nrf2 pathway repressed both to control levels, while Nrf2 deletion annulled this repression, leading to high levels of expression, showing a clear Nrf2-dependent effect. Acacb mitochondrial isoform showed no change (Supplemental Figure 3A). Expression of Acly (Figure 5C) and Pdhal (Figure 5E), which directly lead to increased levels of available acetyl-CoA for lipogenesis, was not altered in AdNICD mice compared with controls, while Me1 expression, which produces pyruvate and NADPH for lipogenesis, increased in AdNICD mice and returned to control levels after Nrf2 pathway activation (Figure 5D). Of the elongases examined (Figure 5, F-H), Elovl6 was induced approximately 5-fold in AdNICD mice, was repressed to control levels in Keap1KD:AdNICD mice, and rebounded back again in the Nrf2-null background (Figure $5 \mathrm{H})$. Transcript expression of desaturases $S c d 1$ and $S c d 2$ followed similar trends, with 2.5 -fold and 
A

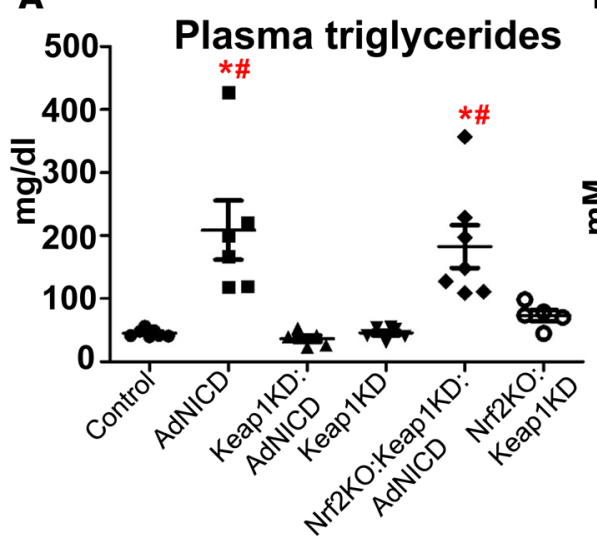

D

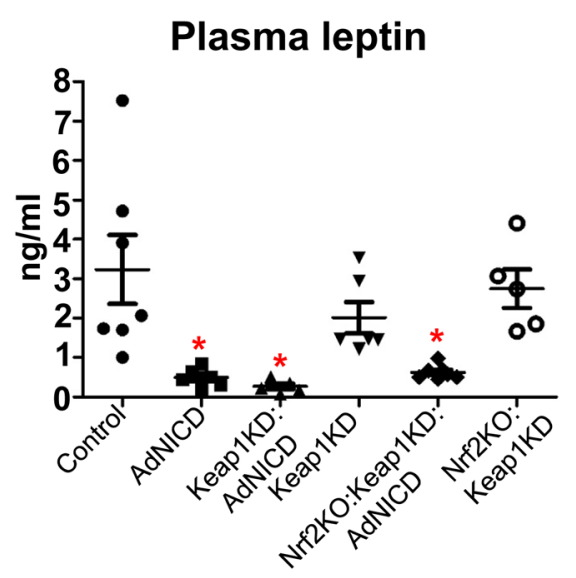

B

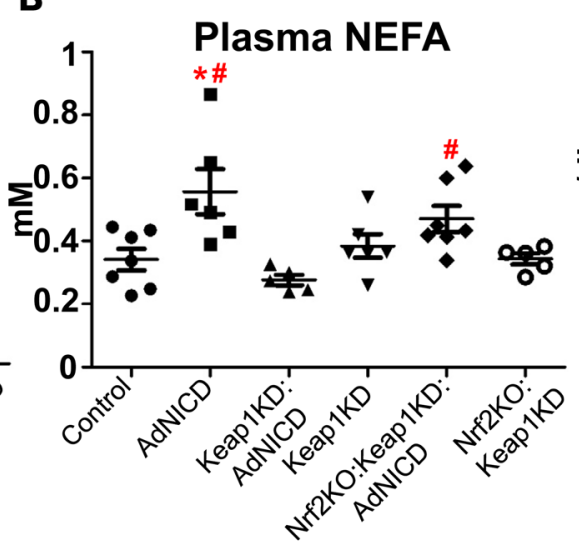

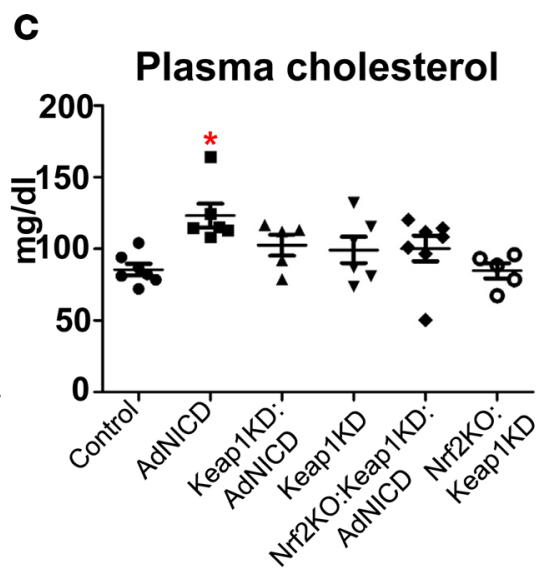

E
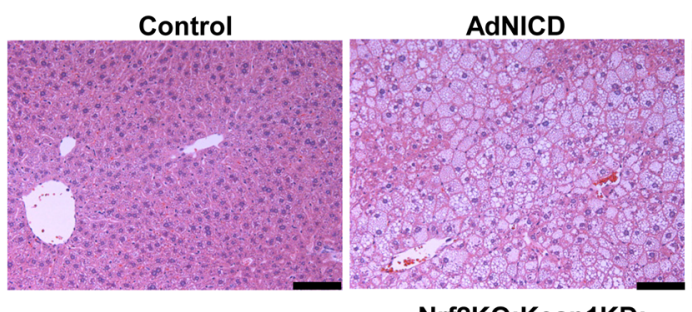

Keap1KD:AdNICD

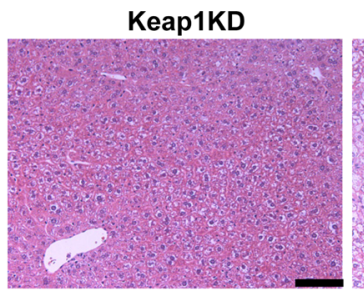

Nrf2KO:Keap1KD:

AdNICD

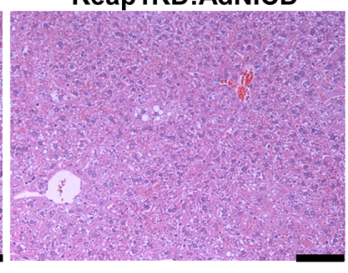

Nrf2KO:Keap1KD
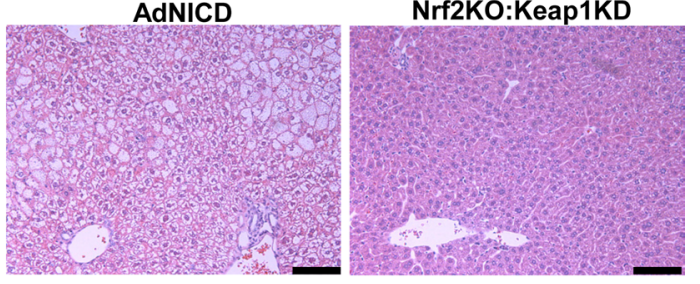

Figure 3. Nrf2 prevents AdNICD mice from dyslipidemia and fatty liver disease. (A) Plasma triglycerides, (B) nonesterified fatty acids (NEFA), (C) cholesterol, and (D) leptin levels measured in 3-month-old male mice. (E) Representative images of H\&E-stained sections of livers of 3-month-old mice. Scale bar: $100 \mu \mathrm{m}$. All data represent mean $\pm \mathrm{SEM}$. ${ }^{*} P<0.05$ compared with control mice; ${ }^{\#} P<0.05$ compared with Keap1KD:AdNICD mice, 1 -way ANOVA.

2-fold increases, respectively, in AdNICD mice and a return to control levels in Keap1KD:AdNICD mice (Figure 5, I and J). Transcript levels of factors that regulate the expression of most of these lipogenic enzymes did not show any major changes (Supplemental Figure 3, B-F). Cd36, which regulates fatty acid uptake, increased in response to the fatty livers of AdNICD mice (Figure $5 \mathrm{~K}$ ). Nrf2 mRNA was absent in the Nrf2-null background as expected and, interestingly, was increased by approximately $60 \%$ in AdNICD mice (Figure 5L), perhaps as a failed compensatory response. A prototypical Nrf2 target gene, Nqo1, was induced approximately 2.3-fold in AdNICD mice and by even more in Keap $1 \mathrm{~K}$ D:AdNICD mice ( 4.6-fold), as expected (Figure 5M). Fgf21, a hormone that has insulin-sensitizing properties but whose levels are increased in insulin resistance, showed increased hepatic expression (Figure $5 \mathrm{~N}$ ) and circulating levels (Figure 5O) in AdNICD mice. No differences were observed in triglyceride biosynthesis (Supplemental Figure 3, G and H), fatty acid oxidation (Supplemental Figure 3, I and J), and gluconeogenic pathways (Supplemental Figure 3, K and L).

Nrf2 pathway activation restores the fatty acid composition of liver triglycerides in AdNICD mice to control levels. Based on the differential expression of genes involved in lipogenic pathways (summarized and highlighted in Figure 6A), we hypothesized that the hepatic triglycerides of AdNICD mice would contain higher amounts of desaturated fatty acids $18: 1$ and 16:1 and would be reversed in Keap1KD:AdNICD mice. Indeed, total levels of 16:1 and 18:1 were higher in AdNICD mice (Figure 6B) as well as the percentage composition of these fatty acids within the triglycerides (Figure 6C) and the ratios 16:1/16:0 and 18:1/18:0 (Figure 6D). Most importantly, these changes in fatty acid composition were 

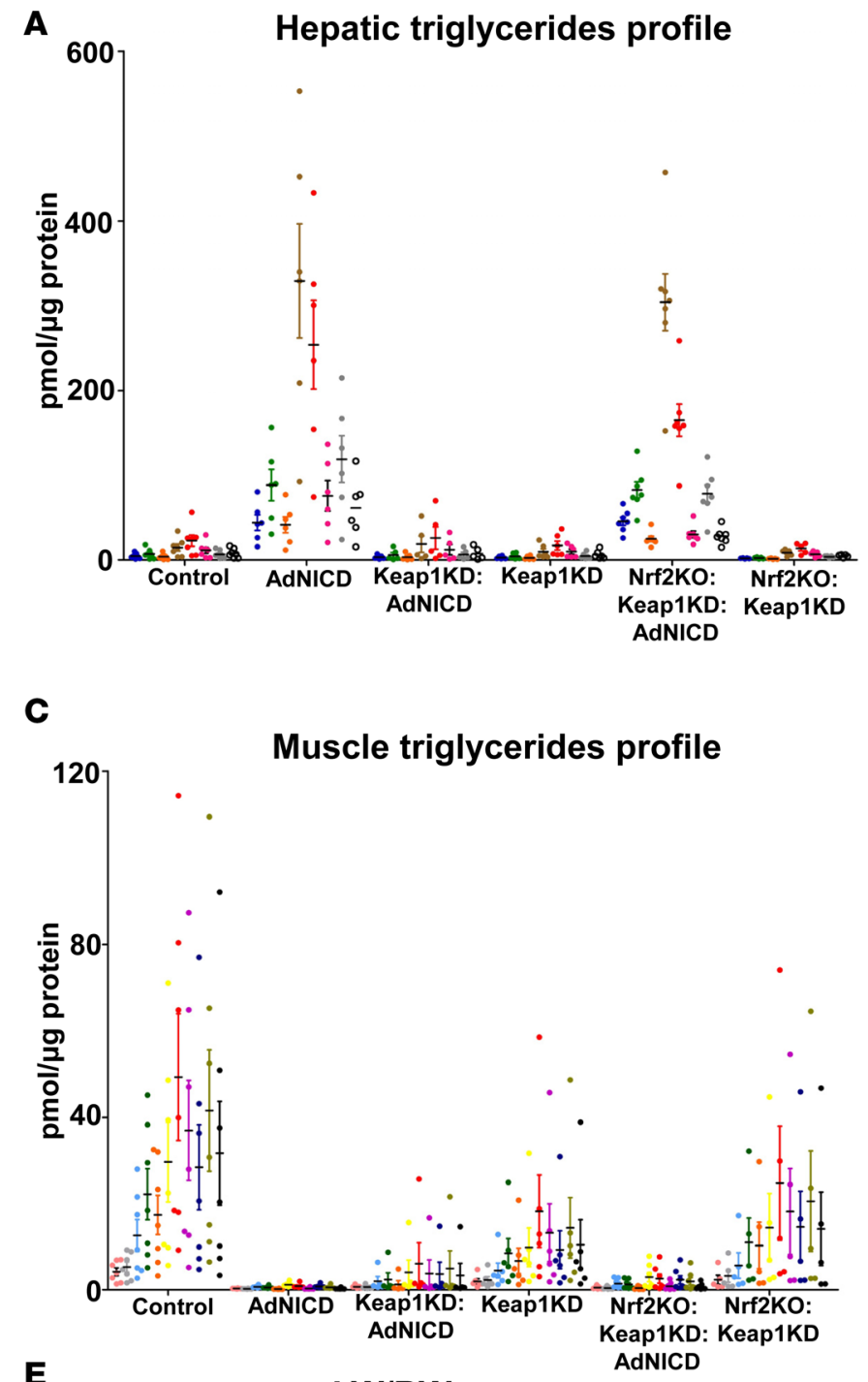

$.48: 1$
$.48: 2$
$.50: 1$
$.50: 2$
$.50: 3$
$52: 2$
$.52: 3$
$.52: 4$
$.54: 3$
$.54: 4$
$.54: 5$

B

\section{Hepatic triglycerides}

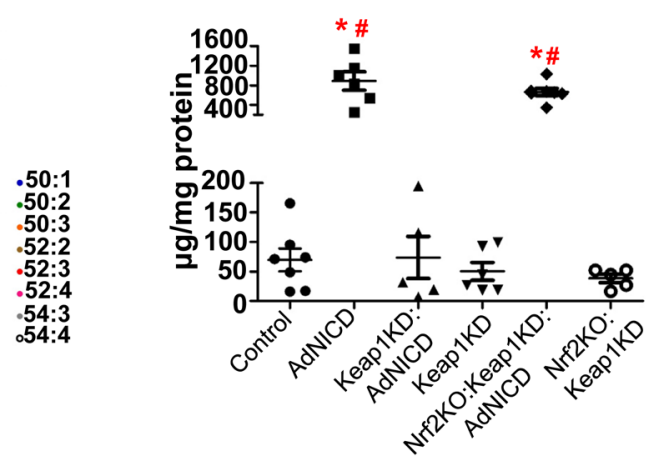

D

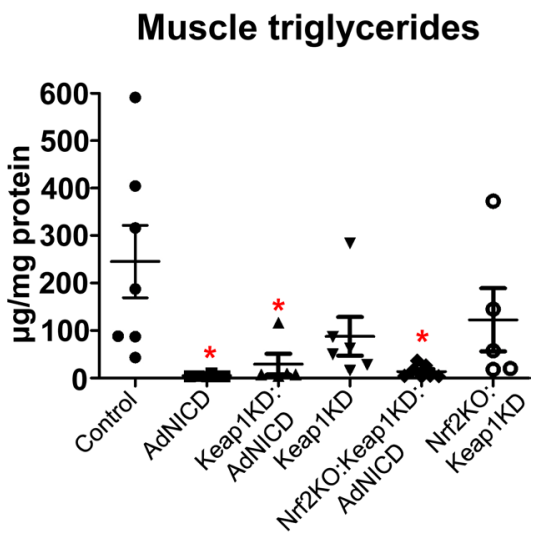

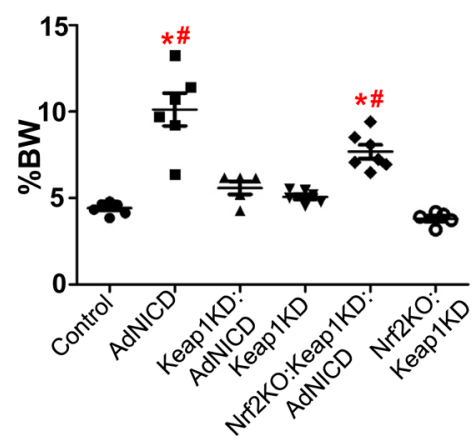

Figure 4. Nrf2 prevents accumulation of triglycerides in the livers of AdNICD mice but has no effect on the muscle. (A) Profiles of hepatic triglycerides species, as assessed by HPLC-MS. (B) HPLC-MS-based total hepatic triglyceride measurement. (C) Profile of muscle triglycerides species, as assessed by HPLC-MS. (D) HPLC-MS-based total muscle triglyceride assessment of 3-month-old mice. (E) Liver-to-body-weight (LW/BW) ratio in 3-month-old male mice. All data represent mean \pm SEM. ${ }^{*} P<0.05$ compared with control mice; ${ }^{\#} P<0.05$ compared with Keap1KD:AdNICD mice, 1-way ANOVA.

abrogated after Nrf2 activation, showing that the fatty acid profiles were likely predicated on the gene expression changes.

Adipocyte-specific Rbpjk deletion in AdNICD mice rescues lipodystrophy and the concomitant insulin resistance and hypertriglyceridemia. As it has been evident, Nrf2 pathway activation through the introduction of the Keap1KD allele into AdNICD mice effectively abrogated lipodystrophic diabetes by completely preventing fatty liver dis- 
A
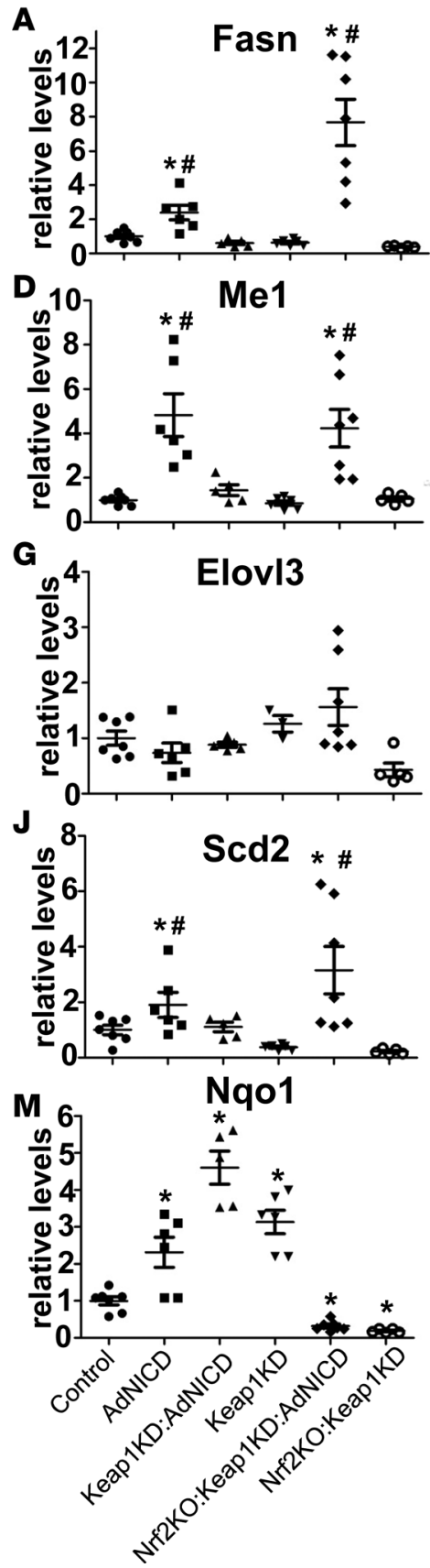
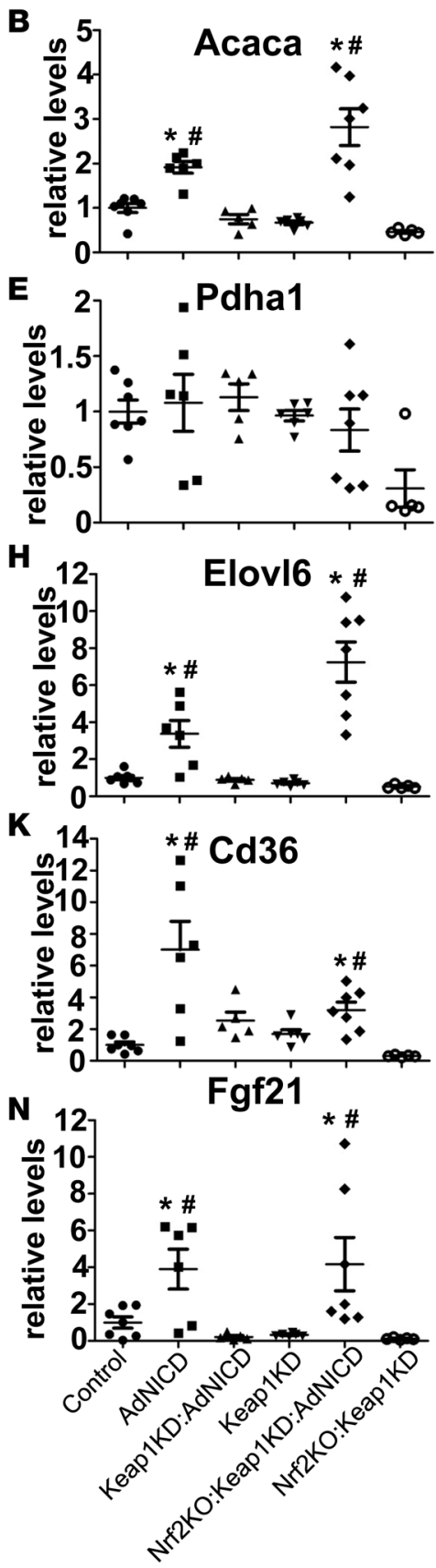
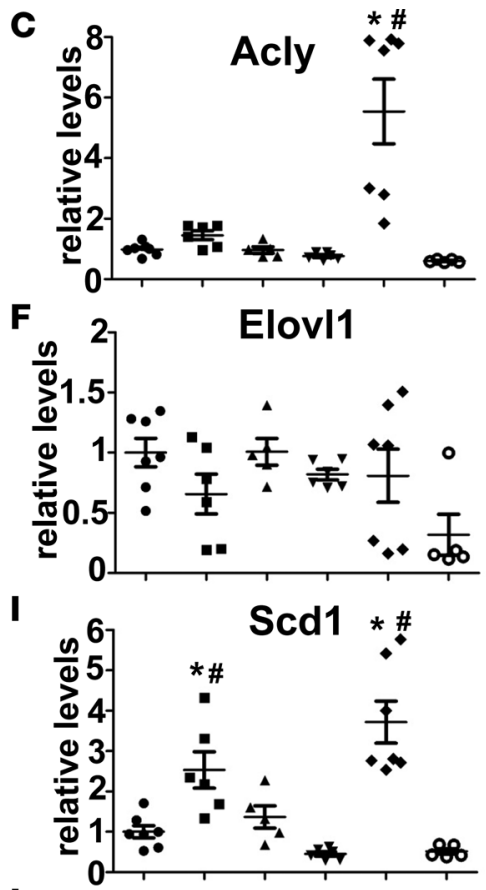

L 2.5
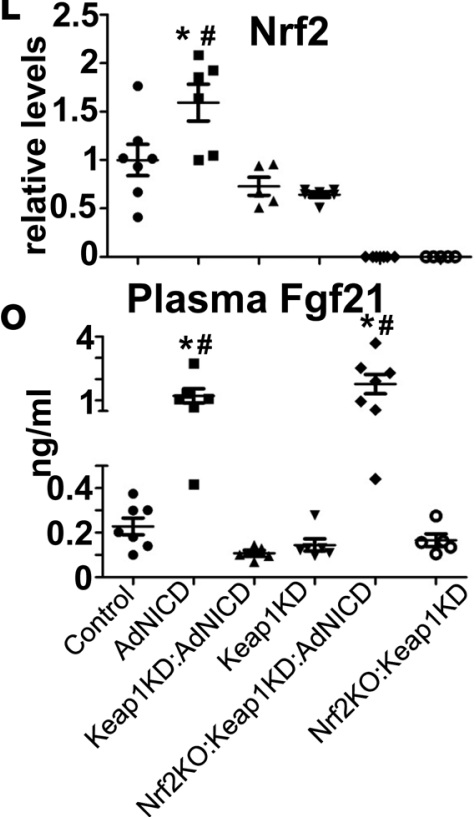

Figure 5. Nrf2 represses lipogenic gene expression in the livers of AdNICD mice. Quantitative real-time PCR for liver mRNA levels of (A and B) the major lipogenic enzymes Fasn and Acaca, (C-E) acetyl-CoA-generating enzymes Acly, Me1 and Pdha1,(F-H) fatty acid elongation enzymes Elov/1, Elov/3, and Elovl6, (I and J) fatty acid desaturation enzymes Scd1 and Scd2, (K) fatty acid translocase Cd36, (L) Nrf2, (M) prototypical Nrf2 target gene Nq01, and (N) metabolic regulator Fgf21. (0) Circulating Fgf21 levels measured with ELISA. Three-month-old male mice were used. All data represent mean \pm SEM. ${ }^{*} P<0.05$ compared with control mice; ${ }^{*} P<0.05$ compared with Keap1KD:AdNICD mice, 1-way ANOVA.

ease through repression of lipogenesis. However, the fate of the adipose tissue remained unchanged, as manipulating Nrf2 did not affect lipodystrophy per se. This is photographically depicted in Figure 7A, with epidydimal white adipose tissue clearly evident in control mice but absent in both AdNICD and Keap1KD:AdNICD mice. To assess the extent to which the diabetic phenotype of AdNICD mice can be prevented by rescuing lipodystrophy per se, we hypothesized that adipocyte-specific deletion of Rbpjk, the principal DNA-binding partner of NICD, would block the actions of AdNICD and allow adipocyte differentiation and expansion of adipose tissue. Indeed,

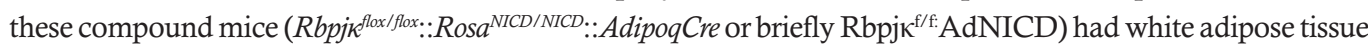
that expanded normally, similar to the control, as it is depicted in Figure 7A (epidydimal white adipose tissue) 
A

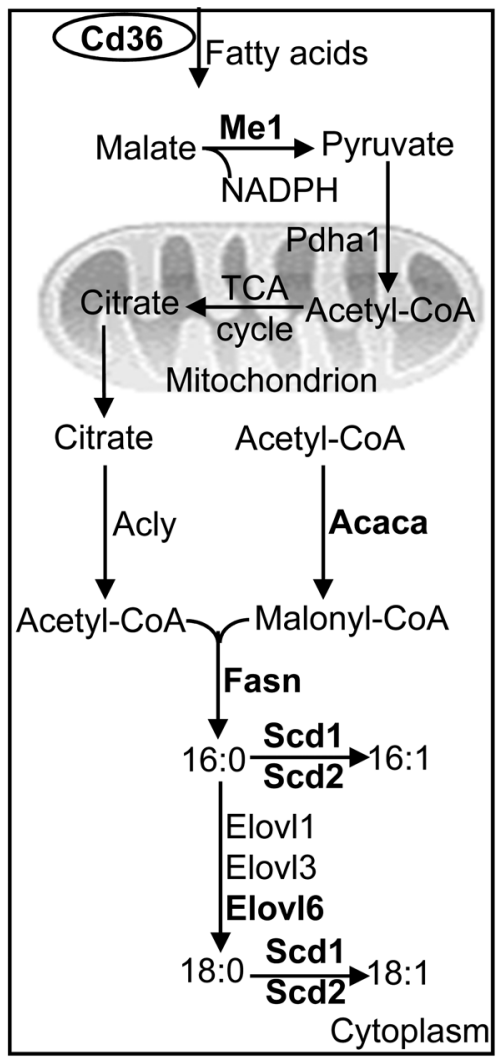

B

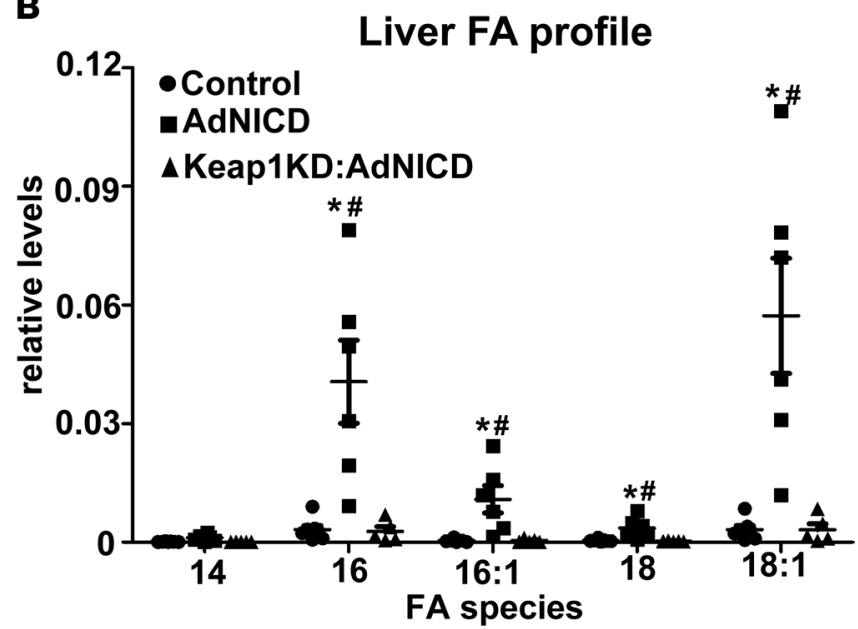

C

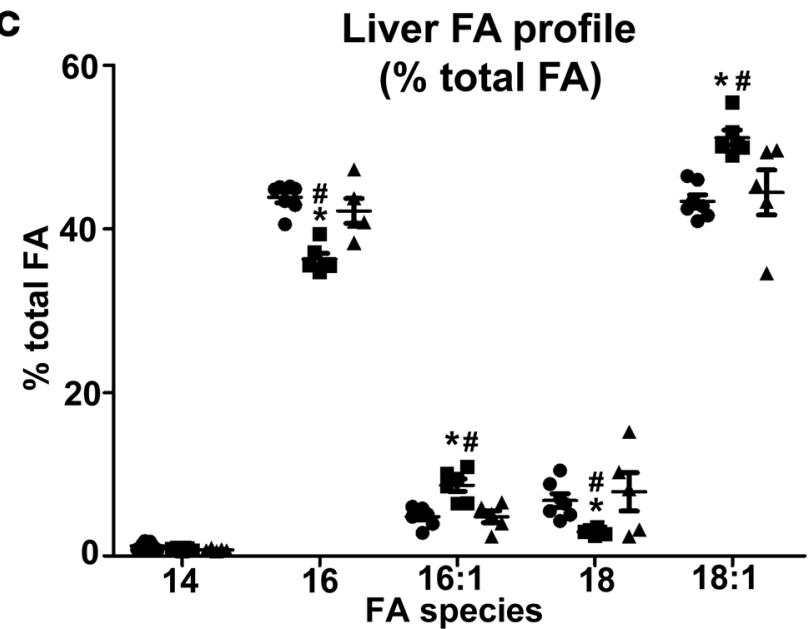

D

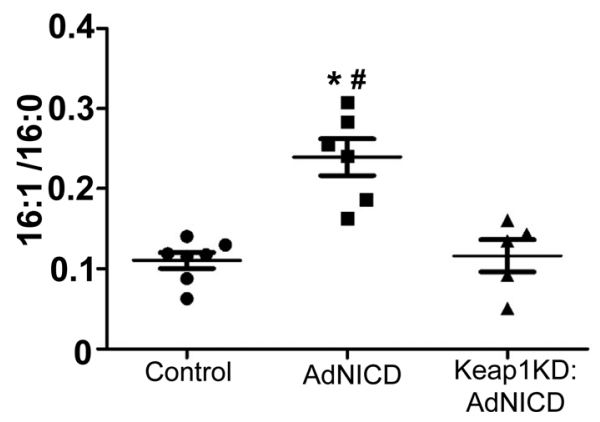

Scd1 desaturation index

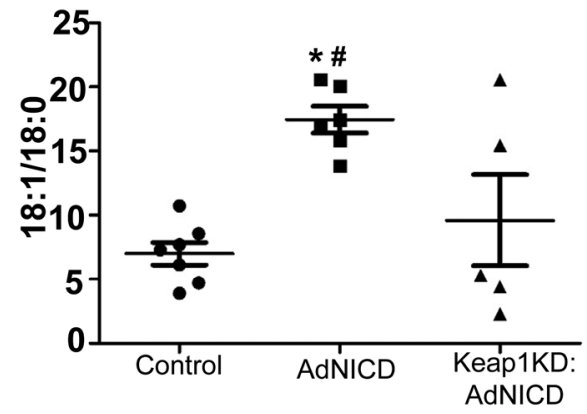

Figure 6. Nrf2 alters the fatty acid composition of hepatic triglycerides. (A) Schematic presentation of the reactions catalyzed by the enzymes. Enzymes upregulated in AdNICD mice and repressed in Keap1KD:AdNICD mice are shown with bold letters. Scheme partially designed using ePath3d. (B) Profile of hepatic fatty acid (FA) species assessed by HPLC-MS, expressed as relative levels. (C) Profile of hepatic fatty acid species assessed by HPLC-MS, expressed as the percentage of the total amount of the measured fatty acids per animal. (D) Ratios of the relative levels of the fatty acid species 16:1/16:0 and 18:1/18:0 that represent ratios of product to precursor fatty acids for the Scd1 enzyme. Three-month-old male mice were used. All data represent mean \pm SEM. ${ }^{*} P<0.05$ compared with control mice; ${ }^{\#} P<0.05$ compared with Keap1KD:AdNICD mice, 1-way ANOVA (D) and 2-way ANOVA (B and C).

and Figure 7B (body fat, as assessed by NMR-based technology). The circulating levels of the adipokine leptin, which were severely repressed in the lipodystrophic AdNICD mice, returned as expected to control levels with the deletion of Rbpjk (Figure 7C). The reinstatement of plasma triglycerides and insulin to control levels (Figure 7, D and E, respectively) is also indicative of the prevention of diabetes and dyslipidemia by rescuing lipodystrophy. An insulin sensitivity test also revealed that insulin resistance was overcome (Figure 7F and Supplemental Figure 4A) through rescue of lipodystrophy. Finally, hepatic steatosis was totally prevented in 
A

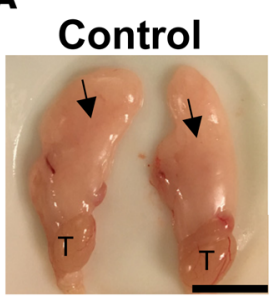

AdNICD

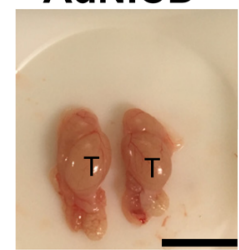

Keap1KD: AdNICD
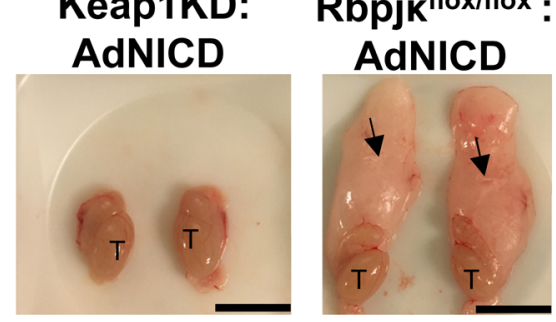

C Plasma leptin

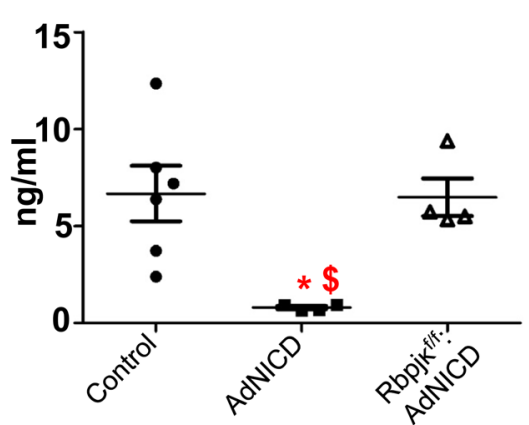

F

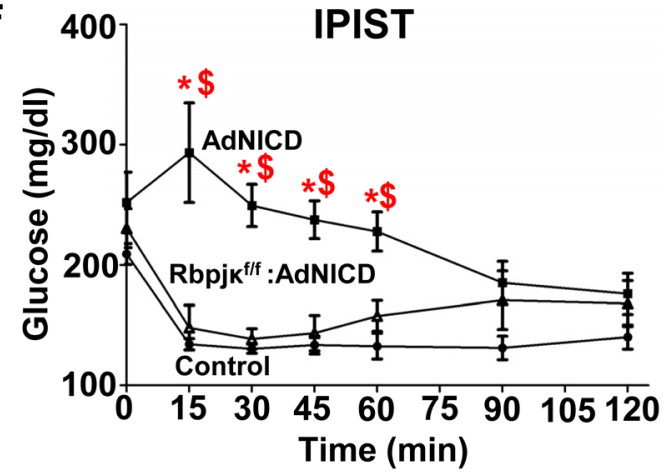

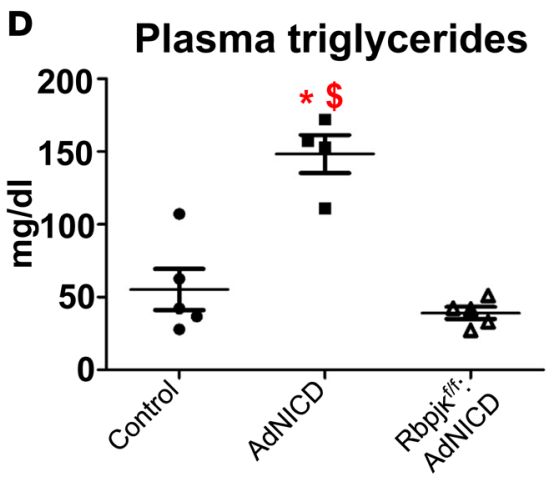

G

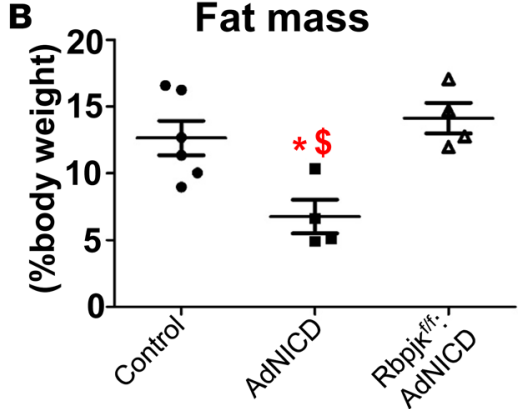

E

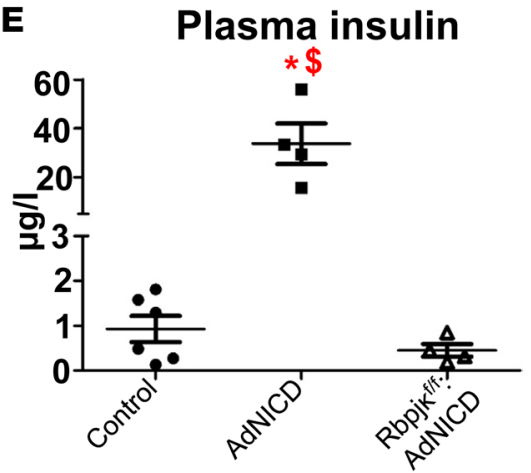

H

Control
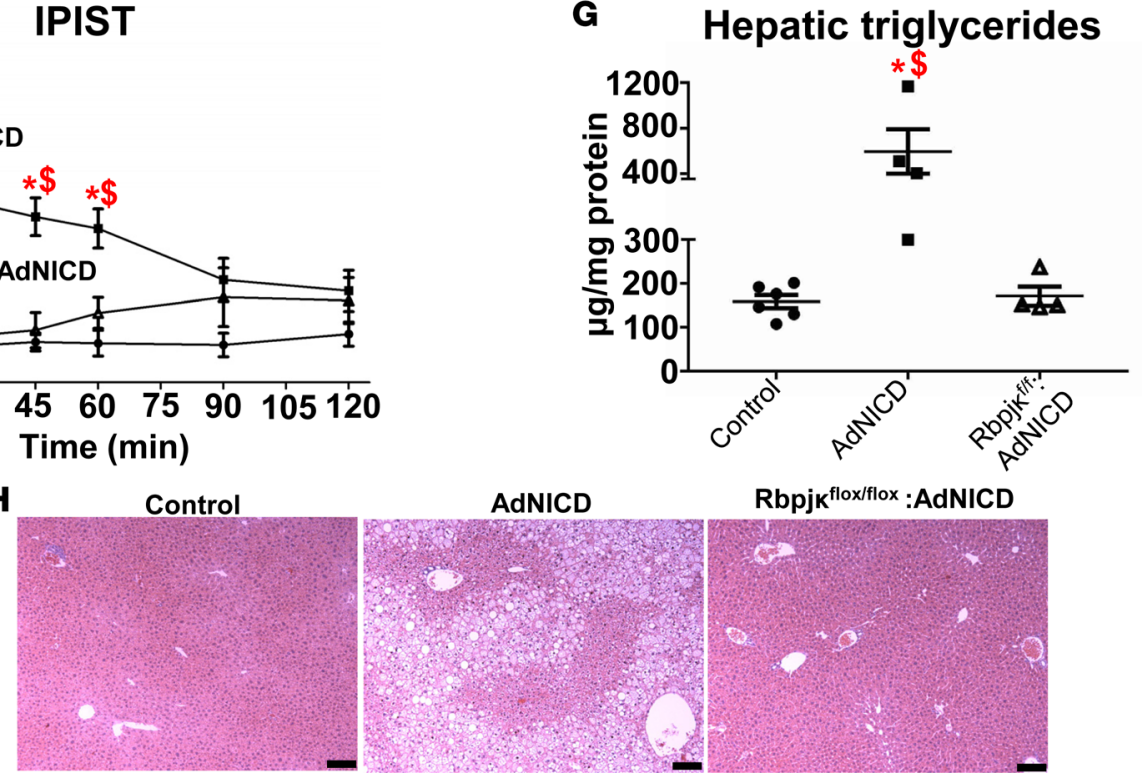

Figure 7. Adipocyte-specific Rbpjк deletion in AdNICD mice restores adipose tissue and rescues lipodystrophic diabetes. (A) Necropsy photos of excised gonadal fat pads from 3-month-old male mice. "T" indicates testis, and the arrows point to the epididymal white adipose tissue. Scale bar: $1 \mathrm{~cm}$. (B) Quantitative magnetic resonance-based measurement of fat mass. Plasma (C) leptin, (D) triglycerides, and (E) insulin in male 3-month-old mice. (F) Blood glucose levels during an intraperitoneal insulin sensitivity test (IPIST) in 2.5-month-old male mice. Control $(n=6)$, AdNICD $(n=4)$, and Rbjא ${ }^{f / f}$ :AdNICD ( $n$ =4). (C) Hepatic triglycerides levels assessed by colorimetry in male 3-month-old mice. (H) Representative images of H\&E-stained sections of livers of 3-month-old male mice. Scale bar: $100 \mu \mathrm{M}$. All data represent mean $\pm \mathrm{SEM} .{ }^{*} P<0.05$ compared with control mice; ${ }^{\$} P<0.05$ compared with Rbj ${ }^{f / f}$ : AdNICD mice, 1-way ANOVA (B-E) and 2-way ANOVA (F).

$\mathrm{Rbpjc}^{\mathrm{f} / \mathrm{f}} \mathrm{AdNICD}$ mice, as is evident from the hepatic liver triglycerides (Figure $7 \mathrm{G}$ ) and the liver sections (Figure 7H).

Nrf2 reduces the incidence and delays the development of Notch-driven sarcomas. To verify that Nrf2 prevented diabetes over the long term, a cohort of aged Keap1KD:AdNICD mice was observed to maintain normoglycemia, with only a mild increase in insulin levels (Figure 8, A and B), while leptin levels remained severely repressed, as expected (Figure 8C). Moreover, we report that at a median age of 225 days (7.5 months) (Figure 8D) nearly all of the AdNICD mice developed tumors (Figure 8E), while Keap1KD:AdNICD mice devel- 

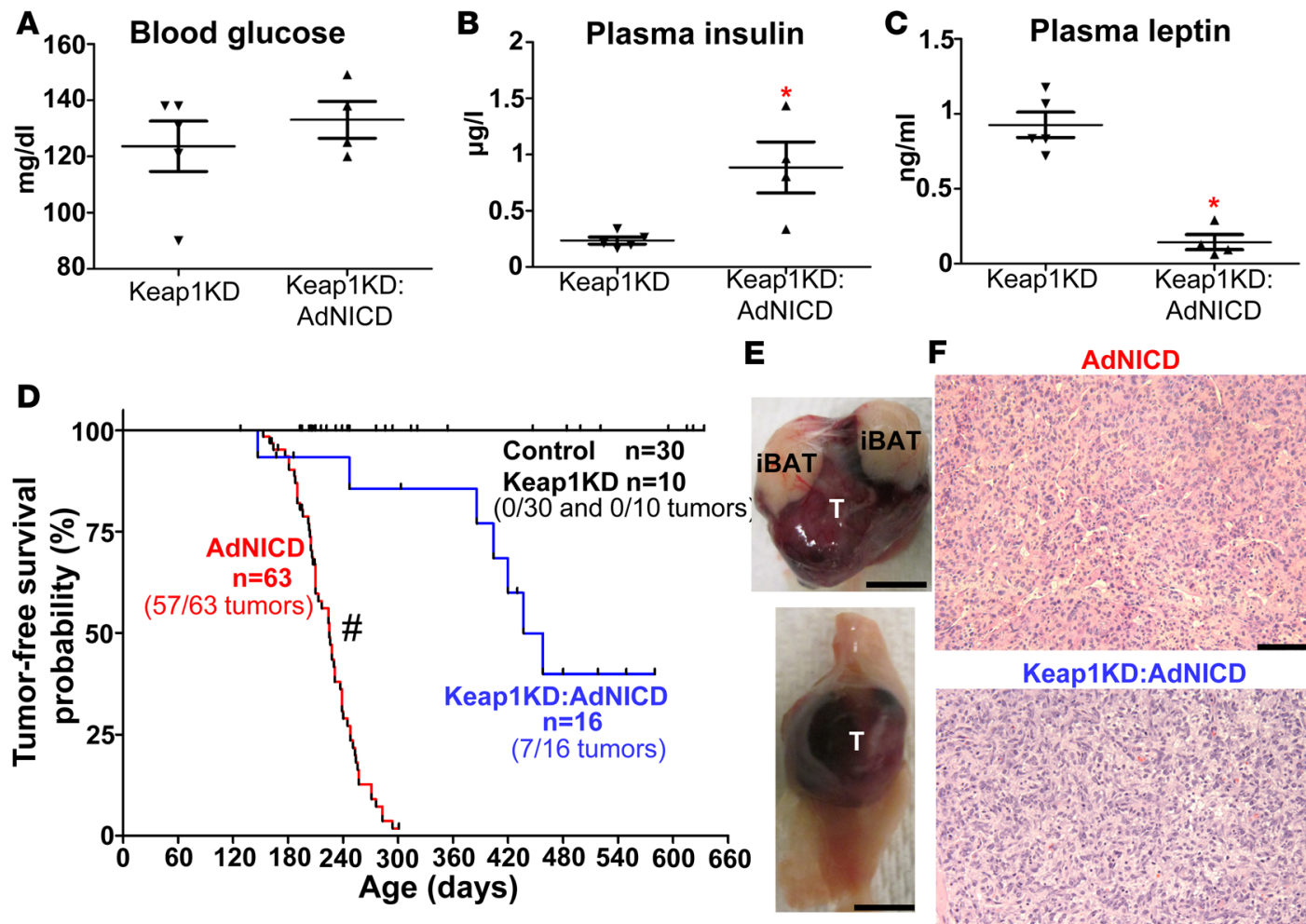

F $\quad$ AdNICD
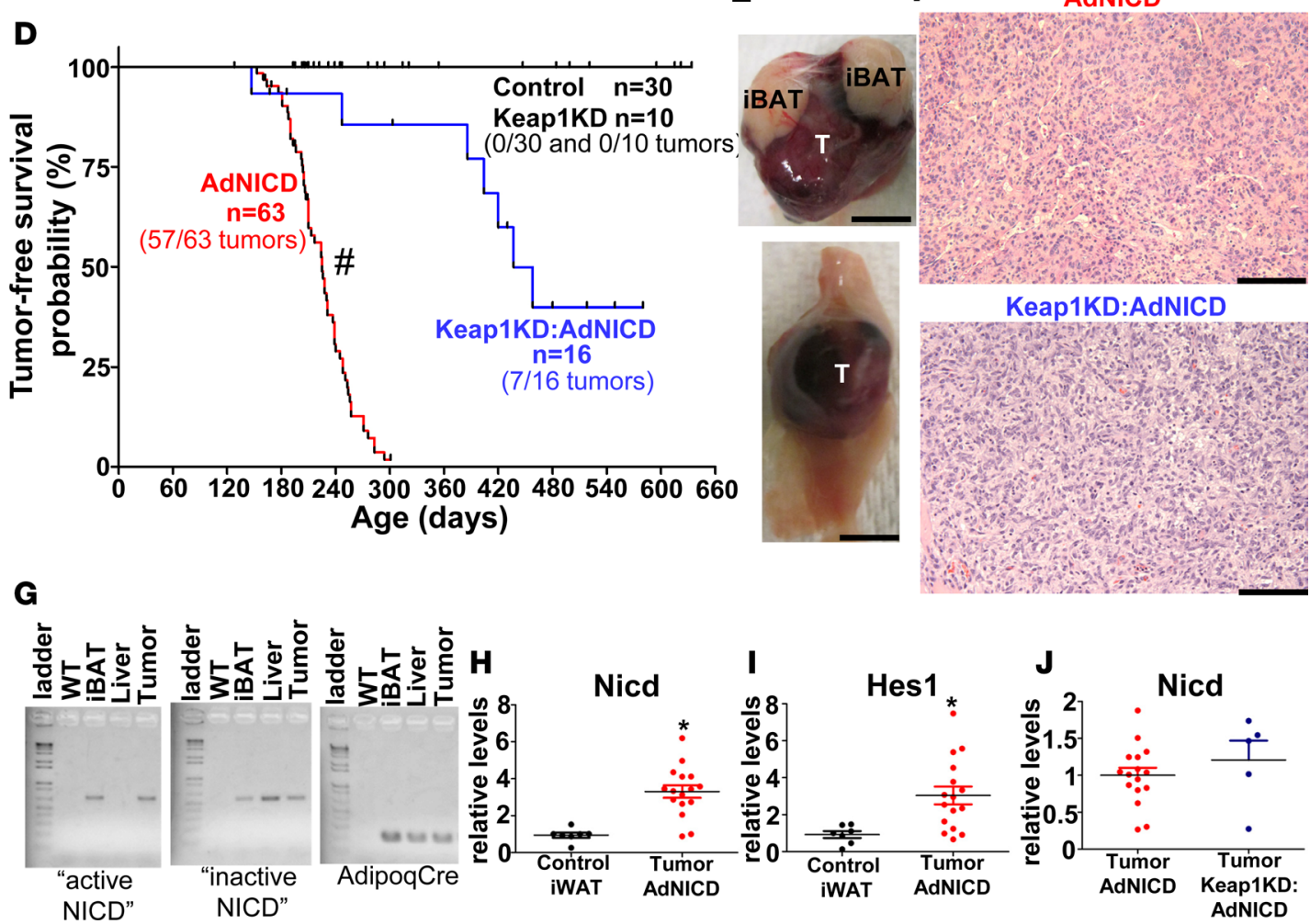

Figure 8. Activation of Nrf2 pathway significantly prevents and delays the onset of sarcomas in AdNICD mice. Circulating (A) glucose, (B) insulin, and (C) leptin levels in aged male Keap1KD and Keap1KD:AdNICD mice (11-14 months old). ${ }^{*} P<0.05, t$ test. (D) Kaplan-Meier survival curve of control, Keap1KD, AdNICD, and Keap1KD:AdNICD male mice. The actual number of tumors detected per genotype is reported in parentheses. ${ }^{*} P<0.0001$ compared with Keap1KD:AdNICD, log-rank test. (E) Photograph of an excised interscapular mass detected in a 7-month-old AdNICD mouse (top) and of an inguinal tumor detected in a 7.5-month-old mouse (bottom). Scale bar: $5 \mathrm{~mm}$. (F) Representative images of H\&E-stained sections of tumors from AdNICD (7-month-old) and Keap1KD:AdNICD (14-month-old) mice. Scale bar: $100 \mu \mathrm{m}$. (G) Representative images of ethidium bromide-stained $2 \%$ agarose gels with PCR products that show the presence or absence of RosaNICD recombination ("active NICD"), the presence of the RosaNICD locus in the genome ("inactive NICD), and the presence of AdipoqCre in the genome (AdipoqCre) in genomic DNA from interscapular brown adipose tissue (iBAT) that serves as a positive control for recombination from liver and from tumor in a 7-month-old male AdNICD mouse. Tail wild-type mouse DNA serves as a negative control. Quantitative real-time PCR of mRNA levels of Nicd (H) and Hes1 (I) in tumors of AdNICD mice and from inguinal white adipose tissue (iWAT) of age-matched control mice. Data represent mean $\pm \mathrm{SEM}, n=7$ for control iWAT and $n=16$ for AdNICD tumors; ${ }^{*} P<0.05, t$ test. (J) Quantitative real-time PCR of mRNA levels of Nicd in tumors of AdNICD and Keap1KD:AdNICD mice. Data represent mean \pm SEM, $n=16$ for AdNICD tumors, $n=5$ for Keap1KD:AdNICD tumors. Significance was assessed using the $t$ test.

oped tumors at almost double that age (437 days or $\sim 14.5$ months) (Figure $8 \mathrm{D}$ ) and at a much lower incidence $(P<0.0001)$. The tumors of both genotypes appear similar, have the characteristics of poorly differentiated high-grade sarcomas (Figure $8 \mathrm{~F}$ ), and are derived by NICD overexpression in adipocytes, as they carry the NICD recombination (Figure $8 \mathrm{G}$ ) and overexpress Nicd (Figure $8 \mathrm{H}$ ) and its target gene Hes1 (Figure 8I). Similar levels of Nicd mRNA were measured in AdNICD and Keap1KD:AdNICD sarcomas (Figure 8J), showing that the expression of the transgene was not affected by Keap1 knockdown. The sarcomas were mostly located in subcutaneous areas of the mice (Figure 9A), and their pathological evaluation by immunostaining revealed an absence of an epithelial nature (negative pankeratin), a lack of endothelial (Cd34 negative) and myogenic components (desmin negative), but S100 positivity to a variable degree (Figure 9B), which is 
A

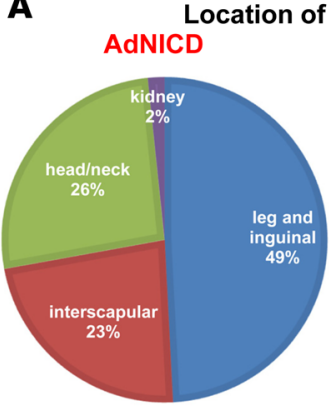

Location of tumors

Keap1KD:AdNICD

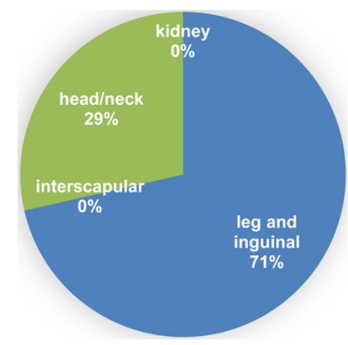

C

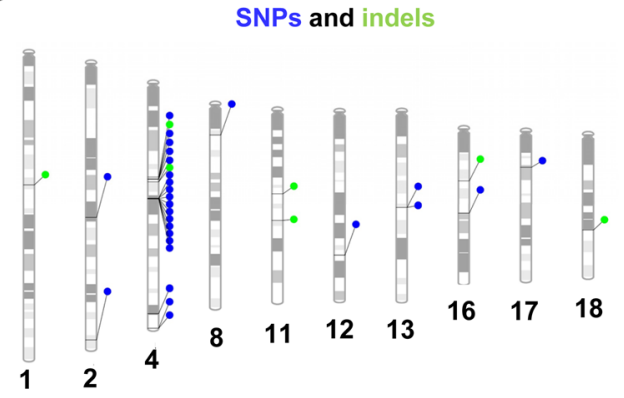

B

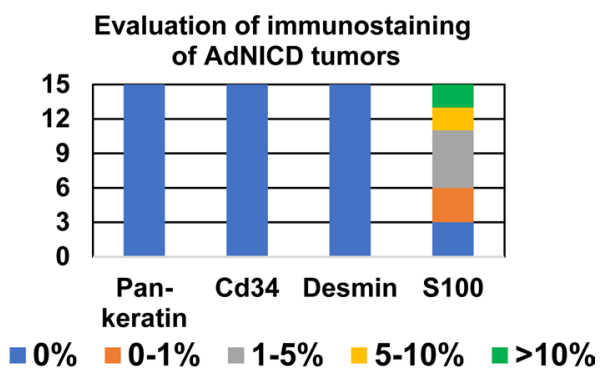

D

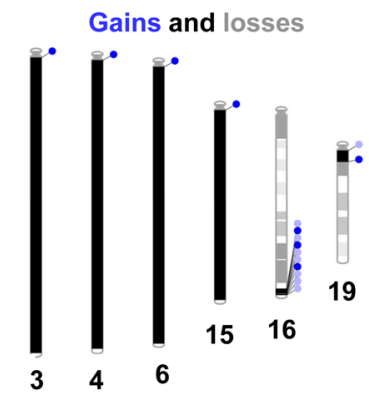

Figure 9. NICD-driven soft-tissue sarcomas show the pathology signature of liposarcomas and increased genomic instability. (A) Location of tumors in AdNICD and Keap1KD:AdNICD mice, expressed as percentages of the total number of tumors. $n=61$ AdNICD (4 mice had 2 parallel tumors); $n=7$, Keap1K$\mathrm{D}:$ AdNICD. All tumors were detected in subcutaneous areas of the mice, with the exception of 1 in the AdNICD mouse, which was located in the perirenal area. (B) Evaluation summary of staining of sections of 15 AdNICD tumors with pankeratin, Cd34, desmin, and S100 antibodies. Mice bearing these tumors had a median age of 7 months. (C) Mapping on the chromosomes of highly significant represented SNPs and indels $(P<0.005)$ among the 5 tumor samples compared with their matched nontumor controls (tails). The "lollipops" indicate their location on the chromosome. Blue lollipops indicate SNPs and green lollipops indicate indels. Only the chromosomes where SNPs or indels were detected are shown. (D) Summary of the overlapping gains (blue lollipops) and losses (gray lollipops) that were detected in at least 4 tumor samples, as determined by analysis of copy number variation (CNV), compared tumor with nontumor-matched controls (tail) using exome sequencing. The thick black line on the chromosome indicates the relative size (length) of each detected CNV. CNVs on chromosomes $3,4,6$, and 15 were detected in all 5 tumors, while those on 16 and 19 were detected in 4 of 5 tumors. Only gains or losses larger than $1 \mathrm{~kb}$ with probability $\geq 50 \%$ are depicted at their starting position on the chromosome. Only the chromosomes where gains or losses were detected are shown.

indicative of their lipogenic nature (liposarcomas). No other tumors, such as hepatocellular carcinomas due to the long-term fatty liver disease, were detected in these mice.

Notch-induced sarcomas show genomic instability. To genetically approach the nature of these liposarcomas developing in AdNICD mice, exome sequencing was performed in 5 of these sarcomas. Genomic DNA from the tails (considered nontumor tissue) of these mice was used as control. Our analyses revealed that these liposarcomas showed genomic instability, as evidenced by the number of SNPs and indels detected (Supplemental Figure 4, B-D). The most highly significant $(P<0.005)$ SNPs and indels are depicted by their relevant chromosomal location in Figure $9 \mathrm{C}$, and the ones affecting coding regions of genes are outlined in Supplemental Tables 2 and 3. To the extent that exome sequence analysis allows evaluation through copy number variation (CNV) analysis, larger chromosomal changes were also revealed. Specifically, as shown in Figure 9D, the CNVs (duplications) in chromosomes 3, 4, 6, and 15 were detected in all 5 sarcoma versus tail comparisons and refer to chromosomal areas larger than $100 \mathrm{Mb}$. The CNVs depicted in chromosomes 16 and 19 were detected in 4 of 5 sample pair-wise comparisons and refer to regions ranging from $1 \mathrm{~Kb}$ to $5 \mathrm{Mb}$. Such genomic DNA aberrations are common in human soft-tissue sarcomas as well (30).

\section{Discussion}

A role for Nrf2 in protecting from insulin resistance due to loss of adipose tissue capacity by repressing hepatic lipogenesis. Nrf2, by exerting its classic cytoprotective actions, can prevent the detrimental effects of chronic oxidative and electrophilic stresses in the setting of obesity and type 2 diabetes, as has been established in the pancreas (31) and hypothalamus (32). However, Nrf2 appears to have multifaceted roles in metabolism through its interaction with other pathways. Herein, we provided evidence that Nrf2 is able to profoundly 
protect against a metabolic cascade that starts with reduced or diminished adipose tissue capacity and leads to severe insulin resistance and diabetes due to ectopic lipid accumulation in liver.

Reduction or loss of adipose tissue capacity can occur in obesity or in nonobese subjects with a predisposition to reduced capacity. To model this pathological state, we featured lipodystrophy, which is characterized by complete or partial lack of adipose tissue due to genetic mutations (congenital) or can be acquired as in the antiretroviral-treated $\mathrm{HIV}^{+}$patients. To this end, our recently developed AdNICD mice (11) were used to induce partial lipodystrophy, affecting the white adipose tissues by repressing adipogenesis through adipocyte-specific NICD overexpression. The AdNICD mice showed low levels of leptin that can lead to increased absolute amount of food consumption. As they do not have the capacity to store the extra energy they receive from food in their adipose tissue, lipids accumulate in the liver and the mice develop profound hepatic steatosis, which is associated with insulin resistance and diabetes. Their body weights are also increased due to increased liver weights and the increased total lean mass (11), which might be attributed to increased muscle mass because of nonspecific activation of Igf- 1 receptors by the extremely high circulating insulin levels that, in turn, regulate muscle growth (33). The tendency for increased energy expenditure in AdNICD mice (Supplemental Figure 1, D-F) is a feature of other lipodystrophy models as well, such as Agpat $2^{-1-}$ mice (34), and it also occurs in humans with highly active antiretroviral therapy-associated lipodystrophy (35). Disruption of the normal daily excursions of RER (Figure 1F) was not due to disturbed circadian rhythm. Circadian rhythm appeared to remain normal, as evidenced by the activity of the mice (Supplemental Figure 2A). Hence, the disrupted daily RER excursions in AdNICD mice are apparently caused by the severe insulin resistance that does not allow the mice to use the carbohydrates from their food when they are alert during the dark cycle. Therefore, AdNICD mice continuously use fat as their main energy substrate and thus do not show the expected increase in RER during the dark cycle. Such a phenomenon is also observed in Agpat $2^{-/}$lipodystrophic mice (34). This preference for use of fatty acids as energy substrate is also observed in antiretroviral-treated $\mathrm{HIV}^{+}$lipodystrophic patients (35). Besides the phenotypical characteristics (fatty liver, insulin resistance, energy metabolism disturbances) that make the AdNICD mice an ideal study subject from a clinical perspective, the adipocyte-specific enhancement of the Notch pathway that leads to lipodystrophy highlights the importance of Notch signaling in adipogenesis. This mimics the increased Notch signaling observed in the subcutaneous lipodystrophic adipose tissues of $\mathrm{HIV}^{+}$patients treated with antiretrovirals (Supplemental Figure 1, A and B) and in progeria syndrome patients (14). Moreover, increased Notch signaling shows a positive correlation with insulin resistance and nonalcoholic fatty liver disease in humans (36).

Sustained upregulation of the Nrf2 pathway in AdNICD mice was achieved by genetically knocking down Keap1. An important feature of the model is that Nrf2 had no effect on adipogenesis in AdNICD mice, as Keap1KD:AdNICD mice remained lipodystrophic, as shown macroscopically (Figure 7A), by NMR (Figure 1B), and by the circulating leptin levels (Figure 3D). Hence, Nrf2 did not interfere with the principle of our model of reduced adipose tissue capacity but nonetheless totally rescued diabetes and fatty liver disease. By contrast to enhanced Nrf2 signaling, adipocyte deletion of Rbpjк opposes the action of NICD overexpression, leading to no lipodystrophy as well as failure to develop diabetes or fatty liver through a mechanistic corruption of the model. The protective effect mediated through Nrf2 action in the liver did not seem to affect food consumption (Figure 1C) and energy expenditure (Figure 1D) (minimizing the possibility for a central hypothalamic role of Nrf2 in this model) and did not have a major effect on the pancreas, as the islet size (Figure 2D) and insulin secretion (Figure 2B) were just proportional to the peripheral insulin resistance. Muscle, another major peripheral tissue, does not seem to be affected by $\mathrm{Nrf2}$, when considering triglyceride accumulation, and does not appear to be the main culprit for peripheral insulin resistance in AdNICD mice, as triglyceride accumulation was low (Figure 4, C and D). Muscle may compensate for the potential increased input of lipids by oxidizing the fatty acids, as described in lipodystrophic Ppary hypomorphic mice (37), but it is not the focus of the present work, as Nrf2 does not affect muscle triglyceride levels and the AdNICD mice are highly insulin resistant, despite the low levels of muscle triglycerides strongly suggesting that the cause of peripheral insulin resistance lies in the development of profound fatty liver disease. Collectively, these data support the conclusion that the main target of Nrf2 for ameliorating the diabetic phenotype of AdNICD mice is the liver.

The effect of Keap1 knockdown on the liver was so profound that liver steatosis was completely rescued (Figure 3E and Figure 4B). The increased expression of the major lipogenic genes Fasn and Acaca in the AdNICD livers also returned back to control levels, along with the expression of fatty acid elongase Elovl6 
and desaturases $S c d 1$ and $S c d 2$ (Figure 5). It is clearly shown by the fact that deletion of Nrf2 in Keap1KD:AdNICD mice results in total rebound of hepatic steatosis and enzymatic expression back to AdNICD mouse levels, and, in some cases, levels higher than these, that this effect of Keap1KD on steatosis and lipogenic gene expression is exclusively Nrf2 dependent. The fact that deletion of Nrf2 in Keap1KD:AdNICD mice led the expression of some hepatic genes not just back to AdNICD levels but to even higher levels (Figure 5) may have more effects than just abrogating the effects of Keap1KD. For example, as shown in a previous study with Nrf2-knockout mice on high-fat diet (38), lack of Nrf2 can increase susceptibility to ER stress and affect the expression of metabolic enzymes, such as the ones we analyzed in the present work. The exact mechanism through which Nrf2 can repress this set of lipogenic genes remains to be elucidated. Fgf21 shows a similar response, which is also indicative of peripheral insulin resistance. Circulating Fgf21 levels are increased in AdNICD mice, as happens in obese subjects (39) and in humans with HIV treatmentrelated lipodystrophy (40) and with mice and humans with congenital lipodystrophy (41). The expression of Fgf21 in the liver, which is the major source of circulating Fgf21, followed a similar pattern. Restoration of Fgf21 levels to control levels in Keap1KD:AdNICD mice is another measure of improved insulin sensitivity in these mice (Figure 5, $\mathrm{N}$ and $\mathrm{O}$ ).

The upregulation of the lipogenic enzymes, which are highlighted in Figure 6A, in AdNICD mice was clearly reflected in the composition of triglycerides in fatty acids. The 16:1 and 18:1 fatty acids showed a relative abundance in AdNICD liver triglycerides (Figure 6C), and the Scd1 desaturation index (concentration ratios 16:1/16:0 and 18:1/18:0) (Figure 6D) was increased as also happens in humans with nonalcoholic fatty liver disease (42). Activation of the Nrf2 pathway returned these ratios to control levels, as happened with the lipogenic enzymes.

It is evident that activation of the Nrf2 pathway is capable of completely rescuing the diabetic phenotype of AdNICD mice, without rescuing lipodystrophy per se. The restoration of adipogenesis following adipocyte deletion of Rbpjk rescued lipodystrophy in AdNICD mice and led to rescue of the insulin-resistant and diabetic phenotype (Figure 7) at roughly the same rate as Nrf2 in the face of persistent lipodystrophy, highlighting the powerful effect that Nrf2 has against lipodystrophic diabetes.

Amelioration of the diabetic metabolic profile by Nrf2 can prevent tumorigenesis. AdNICD mice develop sarcomas at an incidence of $>90 \%$ by 10 months of age. Insulin resistance in these mice develops at a much younger age ( 1 month old) (11) than that of sarcomas development and then exacerbates and becomes full-blown diabetes with extensive fatty liver disease by 3 months of age (the age of the mice in the present metabolic studies). These Notch-driven sarcomas provided us with an opportunity to study the long-term effect of sustained Nrf2 pathway activation in a model that initially developed diabetes and ultimately developed sarcomas and to directly assess a potential link between improved metabolic outcomes by Nrf2 and cancer prevention.

An epidemiological association between obesity and cancer risk has been established (3), and increased insulin resistance is associated with increased breast cancer mortality (43). Preclinical studies using a mouse that lacks both white and brown adipose tissues, due to transgenic expression of the dominant negative protein A-ZIP/F, which prevents the DNA binding of B-ZIP transcription factors of both the C/ EBP and Jun families, established that, even in the absence of adipose tissue but in the presence of severe insulin resistance, tumorigenesis can occur (44). Chemically induced skin carcinogenesis in the A-ZIP/F mouse or its crossing with breast cancer-susceptible mouse strain C3(1)/T-Ag showed enhanced carcinogenesis compared with controls, making the case that the volume of adipose tissue and adipokines secreted from it are not the enhancer of tumorigenesis, since the mouse is lipodystrophic; rather, insulin resistance is responsible along with other signaling molecules such as Igf. Our AdNICD mouse has the advantage of being a "clean" model that combines both development of insulin resistance and tumorigenesis by overexpression of NICD in adipocytes, without requiring the introduction of other variables (treatments with chemicals or extra genotypes).

The AdNICD mice develop soft-tissue high-grade undifferentiated sarcomas with the characteristics of liposarcomas. These liposarcomas show genomic instabilities, ranging from SNPs and indels to large CNVs. Our whole-exome sequencing approach, which covers approximately $1 \%-2 \%$ of genome, may have not revealed the full spectrum of CNVs and other more major chromosomal aberrations (e.g., cross-chromosome events). However, even with these limitations, we were able to detect significant genomic changes, as also happens in human soft-tissue sarcomas (30). Moreover, as already noted, Notch shows aberrant expression in several human cancers and is also overexpressed in human soft-tissue sarcomas $(45,46)$, 
giving the Notch-overexpressing tumor model further clinical significance. Another interesting aspect that has not been thoroughly studied is the potential relationship of Notch-overexpressing cancers and cancer cachexia, which is mainly manifested as loss of muscle and fat mass. A preliminary study has shown that NICD-overexpressing osteosarcomas have the potential to suppress myogenesis via "transmission" of the enhanced Notch signaling by exosomes (47). Theoretically, they could suppress adipogenesis in a similar way, but further studies and evaluation are warranted.

By activating Nrf2 signaling, AdNICD mice experienced sustained protection against diabetes, and Keap1KD: AdNICD mice developed sarcomas at almost double the age ( 14.5 months) of AdNICD mice ( 7.5 months) and at an incidence of $<45 \%$. It is important to note that the enhancement of the Nrf2 pathway did not affect the expression of NICD (Figure 8J) and thereby directly manipulate the model. Thus, the effect of $\mathrm{Nrf} 2$ on the significant reduction and delay in cancer progression could be attributed to the ameliorated metabolic profile of the Nrf2-activated mice. The possible effect of Nrf2 on the tumor microenvironment through modulation of myeloid-derived suppressor cells (48) should be considered as well, but the effect of Nrf2 on the metabolic phenotype is so profound that it likely overshadows any other contributing factors.

In summary, we show that activation of the Nrf2 pathway is an effective means to profoundly ameliorate insulin resistance and completely prevent fatty liver and dyslipidemia in a model of lipodystrophic diabetes. This broad protective effect of the Nrf2 pathway is driven by repressing hepatic lipogenesis, as is evident by effects on hepatic gene expression and the profile and levels of fatty acids. Importantly, Nrf2 activation almost doubles the age of the occurrence of sarcomas in this model, highlighting not only the efficiency of Nrf2 signaling in cancer, but also associating the ameliorated metabolic profile with delay in cancer progression. Given that Nrf2 is being validated as a druggable target in clinical trials, the use of small-molecule inducers of Nrf2 signaling should gain attention for amelioration of metabolic disease and its resultant enhancement of carcinogenesis.

\section{Methods}

Mouse model. Mice were housed at $22^{\circ} \mathrm{C}, 50 \%$ humidity with a 12 -hour-light/dark cycle and ad libitum access to water and food (Prolab Isopro RMH 3000 5P76 irradiated diet, LabDiet). All mice used in the experiments were males of the albino $\mathrm{C} 57 \mathrm{BL} / 6 \mathrm{~J}$ background $\left(\mathrm{B} 6(\mathrm{Cg})-\mathrm{Tyr}^{\mathrm{c}-2 \mathrm{~J} / \mathrm{J}}\right)$. The mouse genotypes and the relevant

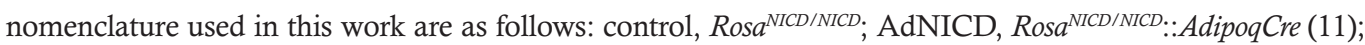

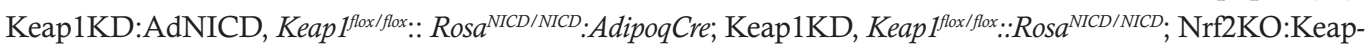

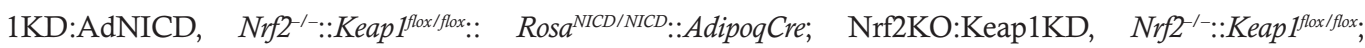

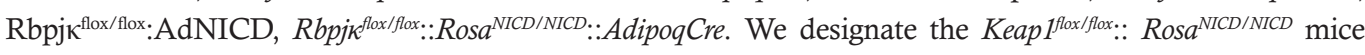
as Keap1 knockdown (Keap1KD), as the loxP site insertion caused substantially less Keap1 expression, as reported above. $N r f 2^{--}$mice (49) and Rbpjkflox/flox mice (50) have been described previously. Breeders for the aforementioned mice were obtained from The Jackson Laboratory, with the exception of Rbpjkflox/fox (Riken)

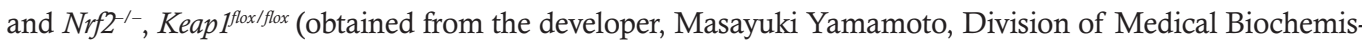
try, Tohoku University School of Medicine, Sendai, Japan), as per the relevant references $(28,49)$. Genotyping PCR primers and primers for the detection of transgenic NICD (Figure $8 \mathrm{G}$ ) and cycling conditions have previously been described $(11,51)$.

Body weight, tissue weights, body composition, and food consumption of mice. Body weight and tissue weights were measured using a precision scale (Scout Pro). Body composition was assessed using EchoMRI model $100 \mathrm{H}$ (EchoMRI). Food consumption was assessed over a 3-day period in single-housed mice.

Indirect calorimetry. Metabolic rate and activity were assessed for a period of 72 hours using the Comprehensive Laboratory Animal Monitoring System (Columbus Instruments).

Intraperitoneal insulin sensitivity test. Insulin sensitivity was assessed by injecting mice that had been fasted for 4 hours with free access to water intraperitoneally with 0.75 units $/ \mathrm{kg}$ insulin (Humalog, Eli Lilly). Blood glucose was measured before the injection with insulin and at the indicated time intervals from tail blood using a Contour next EZ glucose meter (Bayer).

Plasma metabolites. Blood was collected by cardiac puncture during sacrifice under isoflurane anesthesia using heparinized syringes and was kept on ice until centrifugation at 3,000 $\mathrm{g}$ for 30 minutes at $4^{\circ} \mathrm{C}$ for plasma isolation. Insulin was measured using an ELISA kit from Mercodia (catalog 10-1247-01); leptin (catalog MOB00) and Fgf21 (catalog MF2100) were measured using R\&D Systems kits. Triglycerides and cholesterol concentrations were assessed using colorimetric (catalog 10010303) and fluorometric (catalog 10007640) assay kits (Cayman Chemical). Nonesterified fatty acids were measured using the NEFA-HR kit (Wako Chemicals). 
Histology and immunohistochemistry. After dissection, pancreata were immersed in $4 \%$ paraformaldehyde solution (ThermoFisher) in PBS (Life Technologies, ThermoFisher), and liver or tumors were immersed in 10\% buffered formalin (ThermoFisher). After 24 hours, samples were immersed in $70 \%$ ethanol, processed, and embedded in paraffin blocks. Sections were stained with H\&E in the Department of Pathology Development Laboratory at the University of Pittsburgh. Tumors were stained in the same lab with antibodies against Cd34 (catalog 790-2927, Ventana Medical Systems-Roche), desmin (catalog M0760, Dako-Agilent), pan Keratin (catalog ab8068, Abcam), and S100 (catalog Z0311, Dako-Agilent). Pancreata were stained with antibody against insulin (catalog PA1-26938, ThermoFisher). Imaging was performed with either a Leica DM5000B or a Olympus Fluoview 1000 microscope. ImageJ software (NIH) was used to evaluate the insulin-positive area in the pancreatic islets.

Analysis of hepatic and muscle triglycerides. Frozen liver and muscle samples ( $70 \mathrm{mg})$ were thawed on ice and homogenized in a bullet blender (Next Advance) for 5 minutes in $50 \mathrm{mM}$ phosphate buffer, $\mathrm{pH}$ 7.4, containing BHT $(0.16 \%)$. An aliquot of the homogenate $(500 \mu \mathrm{g} / 500 \mu \mathrm{l})$ was spiked with 575 pmol triheptadecanoin (Nu-Check Prep) as internal standard, and lipids extracted using the Bligh and Dyer procedure (52). The organic extracts were dried under nitrogen and reconstituted in ethyl acetate. Triglycerides were analyzed by high-performance liquid chromatography high-resolution mass spectrometry using an analytical C18 Luna column $(2 \times 150 \mathrm{~mm}, 5 \mu \mathrm{m}$, Phenomenex $)$ at a $0.4 \mathrm{ml} / \mathrm{min}$ flow rate using the following gradient program: 35\%-90\% solvent B (0.1-9 minutes), 90\% solvent B (9-11 minutes), followed by 2.5 minutes of reequilibration to initial conditions with a postcolumn infusion of $50 \mu 1 / \mathrm{min} 10 \mathrm{mM}$ ammonium acetate in acetonitrile/water (9:1, v/v). Solvent A was 9:1 acetonitrile/water and solvent B was ethyl acetate. A Q-Exactive hybrid quadrupole-orbitrap mass spectrometer (ThermoFisher) in positive mode was used to quantify the triglycerides species using triheptadecanoin as internal standard. Trihexadecanoin (Nu-Check Prep) standard was used as calibrant for the calibration curve with triheptadecanoin as internal standard. The triglycerides levels were normalized to total protein (Bradford method, Bio-Rad).

Specifically, for determination of total liver triglycerides in Figure 7G, a colorimetric assay kit (catalog 10010303, Cayman Chemical) was used, and triglycerides levels were normalized to total protein.

Analysis of fatty acids in hepatic triglycerides. Lipids were extracted from liver homogenates (10 mg tissue $/ \mathrm{ml}$ ) in the presence of $5.75 \mathrm{nmol}$ triheptadecanoin internal standard using a methanol/dichloromethane extraction (52). Lipids were loaded onto solid-phase extraction Strata NH2 columns (100 mg/1 $\mathrm{ml}$ ), cholesterol esters were eluted first with $2 \mathrm{ml}$ hexane, and triglycerides were then recovered with 2 $\mathrm{ml}$ hexane/chloroform/ethyl acetate (100:5:5, v/v/v) (53). The triglyceride fraction was spiked with 7 $\mu \mathrm{g}$ 16:1-d14, OA-d17, AA-d8 and $70 \mu \mathrm{g}$ 14:0-d27, 16:0-d31, 18:0-d35; dried under a stream of nitrogen; and dissolved in $850 \mu \mathrm{l}$ methanol/dichloromethane (8:1, v/v). Triglycerides were hydrolyzed by adding $150 \mu 1$ of $40 \%$ aqueous potassium hydroxide and heating to $70^{\circ} \mathrm{C}$ for 30 minutes. After acidification using hydrochloric acid, fatty acids were extracted, derivatized to a picolylamide derivatives (54), and quantified by LC-tandem mass spectrometry analysis using a Q-Trap 6500+ mass spectrometer (Sciex) in positive ion mode. Heptadecanoin obtained from the hydrolysis of triheptadecanoin was used as internal standard, and fatty acids were normalized to protein content. Blank samples were processed and background signal was subtracted from samples.

Gene expression. Tissues were homogenized in Trizol (ThermoFisher) using the TissueRuptor homogenizer (Qiagen), and then total RNA was extracted and further purified using the RNeasy kit (Qiagen) after on-column DNAse digestion. RNA was quantified by spectrophotometry using Nanodrop One (ThermoFisher), and purity was assessed by the absorbance ratios of 260:280 and 260:230 nm. RNA integrity was assessed by agarose gel electrophoresis. cDNA was synthesized using the qScript system (Quanta Biosciences). Real-time PCR was performed using an iCycler-MyIQ (Bio-Rad) with iQ SYBR green supermix (Bio-Rad) in triplicate $20 \mu 1$ reactions. The PCR efficiency for each primer pair was determined from standard curves, and if samples were run on more than one 96-well plate, a sample served as interplate calibrator. The generated amplicon was checked by the presence of a single peak in the melting curve analysis. Relative quantification of each target's mRNA levels was performed using the Pfaffl method (55). The stability of 4 potential reference genes was evaluated by using the NormFinder algorithm (56), and the most stable gene was used in the relative quantification calculations ( $R p s 29$ in the case of the liver quantitative PCR and $B 2 m$ in the tumor vs. inguinal white adipose tissue or tumor vs. tumor PCR). Primers sequences are described in Supplemental Table 1; they are mostly derived from the PrimerBank (57) or custom designed using Primer-BLAST (NCBI). 
Exome sequencing, SNP and indel calls, and CNV analysis. Genomic DNA was prepared from 5 tumors of AdNICD mice and 5 tails (considered as normal noncancer tissue) from the same animals using the QIAamp DNA mini kit (Qiagen). DNA was quantified by spectrophotometry using Nanodrop One, and purity was assessed by the absorbance ratios of 260:280 and 260:230 $\mathrm{nm}$. The integrity of the DNA was evaluated by the genomic DNA ScreenTape assay (Agilent) in the Agilent TapeStation system, and the DNA integrity number was calculated for each sample. Next-generation sequencing services were provided by Admera Health LLC. The SureSelect Mouse All Exon Kit (Agilent) was used for library preparation, and the HiSeq sequencing system (Illumina) was used. Read length was $2 \times 150 \mathrm{bp}$ (paired end), with mean on target coverage per sample of $\times 100$. Raw sequence data files (fastq) have been submitted to NCBI Sequence Read Archive under accession SRX3090633-SRX3090642. The Burrows-Wheeler Alignment tool (58) was used to map the exome sequencing reads against the mouse genome (GRCm38/UCSCmm10). SAMtools (59) was used to produce the sequence alignment/map (SAM) files and to convert SAM to BAM files and BAM to pileup files. Varscan (60) was employed to call SNPs and indels from SAMtools pileup files and finally produce the VCF files. Supplemental Figure 4, B-D, was generated using Maftools (61). For statistical analysis of the SNPs and indels among the 5 tumors, VCF files were converted to PLINK files using VCFTools (62). PLINK files were edited to include phenotype data (normal and tumor). Association analysis was performed using PLINK functionality (63), and $P$ values from the association analysis were adjusted for multiple comparisons using all available adjustment methodologies available within "p.adjust" function present in "stats" R library. Figure 9, C and D, which depicts the mapping of SNPs, indels, and CNVs, was generated using Phenogram (64). CNV analysis of tumors was performed by Admera Health LLC by employing a "read depth approach," as the depth of coverage is directly correlated to the copy number of the region. The estimation of CNVs is reported as relative copies to the control normal tissue (mouse tail in our case).

Statistics. Data were analyzed using the Prism version 5 (GraphPad, La Jolla, CA), with the exceptions of the ANCOVA analysis, which was performed using SPSS statistics version 24 (IBM), and the exome sequencing analysis, in which software $\mathrm{R}$ was used. All results are presented as mean $\pm \mathrm{SEM}$. For the comparison of 2 groups, unpaired 2-tailed Student's $t$ test was used; for more than 2 groups, 1- or 2-way ANOVA was used followed by Tukey, as described in the relevant figure legends. The log-rank test was used to compare the survival curves in Figure 8D. Differences with $P$ values of less than 0.05 were considered to be significant. Sample size was estimated on the basis of preliminary studies and previous experimental studies performed in our lab. Experiments were not blinded, with the exception of the intraperitoneal insulin sensitivity test and the histology sections evaluations. No mice were excluded from our analyses.

Study approval. All mice used in this study received humane care that adhered to the principles stated in the Guide for the Care and Use of Laboratory Animals (NIH publication, 1996 edition). The protocol describing all mouse experiments was approved by the Institutional Animal Care and Use Committee of University of Pittsburgh.

\section{Author contributions}

DVC designed and performed all experiments, analyzed data, and wrote the manuscript. YY designed and carried out the pancreatic islets study, analyzed the relevant data, and contributed to all the in vivo experiments. MF designed and performed the HPLC-MS-based assays for triglycerides and fatty acids and analyzed the relevant data. DLP contributed to the in vivo cancer experiments and to the discussion. UNMR designed and carried out the pathology evaluation of the tumors. AZ performed the analysis of the tumor exome sequencing data set. NKHK contributed to the design of in vivo metabolic studies and to the relevant data interpretation. FJS contributed to the design of HPLC-MS-based analysis of lipids and to the discussion. KRW contributed to the in vivo cancer studies and to the discussion. GKM evaluated the liver histology. IS carried out the indirect calorimetry experiment. RMO contributed to design of metabolic studies and to discussion. TWK directed the research and cowrote the paper. NW contributed to design and development of all genetic models and codirected the research.

\section{Acknowledgments}

This work was supported by NIH grants R35 CA197222 (to TWK), R01 DK102839 (to RMO), and P30CA047904. We also acknowledge the support of the Center for Metabolism and Mitochondrial Medicine of the University of Pittsburgh. 
Address correspondence to: Thomas W. Kensler, 200 Lothrop Street, E1352 BST, Pittsburgh, Pennsylvania 15261, USA. Phone: 412.648.1942; Email: tkensler@pitt.edu.

1. Kahn BB, Flier JS. Obesity and insulin resistance. J Clin Invest. 2000;106(4):473-481.

2. Kenchaiah S, et al. Obesity and the risk of heart failure. N Engl J Med. 2002;347(5):305-313.

3. Calle EE, Rodriguez C, Walker-Thurmond $\mathrm{K}$, Thun MJ. Overweight, obesity, and mortality from cancer in a prospectively studied cohort of U.S. adults. N Engl J Med. 2003;348(17):1625-1638.

4. Vigneri P, Frasca F, Sciacca L, Pandini G, Vigneri R. Diabetes and cancer. Endocr Relat Cancer. 2009;16(4):1103-1123.

5. Kershaw EE, Flier JS. Adipose tissue as an endocrine organ. J Clin Endocrinol Metab. 2004;89(6):2548-2556.

6. Lotta LA, et al. Integrative genomic analysis implicates limited peripheral adipose storage capacity in the pathogenesis of human insulin resistance. Nat Genet. 2017;49(1):17-26.

7. Patni N, Garg A. Congenital generalized lipodystrophies--new insights into metabolic dysfunction. Nat Rev Endocrinol. 2015;11(9):522-534.

8. Robbins AL, Savage DB. The genetics of lipid storage and human lipodystrophies. Trends Mol Med. 2015;21(7):433-438

9. Karelis AD, St-Pierre DH, Conus F, Rabasa-Lhoret R, Poehlman ET. Metabolic and body composition factors in subgroups of obesity: what do we know? J Clin Endocrinol Metab. 2004;89(6):2569-2575.

10. Asterholm IW, Halberg N, Scherer PE. Mouse Models of Lipodystrophy Key reagents for the understanding of the metabolic syndrome. Drug Discov Today Dis Models. 2007;4(1):17-24

11. Chartoumpekis DV, et al. Notch intracellular domain overexpression in adipocytes confers lipodystrophy in mice. Mol Metab. 2015;4(7):543-550.

12. Shahmanesh M, Phillips K, Boothby M, Tomlinson JW. Differential adipose tissue gene expression profiles in abacavir treated patients that may contribute to the understanding of cardiovascular risk: a microarray study. PLoS One. 2015;10(1):e0117164.

13. Carr A, et al. An objective case definition of lipodystrophy in HIV-infected adults: a case-control study. Lancet. 2003;361(9359):726-735.

14. Scaffidi P, Misteli T. Lamin A-dependent misregulation of adult stem cells associated with accelerated ageing. Nat Cell Biol. 2008;10(4):452-459.

15. Merideth MA, et al. Phenotype and course of Hutchinson-Gilford progeria syndrome. N Engl J Med. 2008;358(6):592-604.

16. Rosenbloom AL, Kappy MS, DeBusk FL, Francis GL, Philpot TJ, Maclaren NK. Progeria: insulin resistance and hyperglycemia. J Pediatr. 1983;102(3):400-402.

17. Mounkes LC, Kozlov S, Hernandez L, Sullivan T, Stewart CL. A progeroid syndrome in mice is caused by defects in A-type lamins. Nature. 2003;423(6937):298-301.

18. Tao J, et al. Notch activation as a driver of osteogenic sarcoma. Cancer Cell. 2014;26(3):390-401.

19. O'Neil J, et al. FBW7 mutations in leukemic cells mediate NOTCH pathway activation and resistance to gamma-secretase inhibitors. J Exp Med. 2007;204(8):1813-1824.

20. Westhoff B, et al. Alterations of the Notch pathway in lung cancer. Proc Natl Acad Sci USA. 2009;106(52):22293-22298.

21. Motohashi H, Yamamoto M. Nrf2-Keap1 defines a physiologically important stress response mechanism. Trends Mol Med. 2004;10(11):549-557.

22. Uruno A, et al. The Keap1-Nrf2 system prevents onset of diabetes mellitus. Mol Cell Biol. 2013;33(15):2996-3010.

23. Axelsson AS, et al. Sulforaphane reduces hepatic glucose production and improves glucose control in patients with type 2 diabetes. Sci Transl Med. 2017;9(394):eaah4477.

24. Egner PA, et al. Rapid and sustainable detoxication of airborne pollutants by broccoli sprout beverage: results of a randomized clinical trial in China. Cancer Prev Res (Phila). 2014;7(8):813-823.

25. Wakabayashi N, et al. Regulation of notch1 signaling by nrf2: implications for tissue regeneration. Sci Signal. 2010;3(130):ra52.

26. Uruno A, et al. Nrf2-Mediated Regulation of Skeletal Muscle Glycogen Metabolism. Mol Cell Biol. 2016;36(11):1655-1672.

27. Itoh K, et al. Keap1 represses nuclear activation of antioxidant responsive elements by Nrf2 through binding to the amino-terminal Neh2 domain. Genes Dev. 1999;13(1):76-86.

28. Taguchi K, Maher JM, Suzuki T, Kawatani Y, Motohashi H, Yamamoto M. Genetic analysis of cytoprotective functions supported by graded expression of Keap1. Mol Cell Biol. 2010;30(12):3016-3026.

29. Wakabayashi N, et al. Keap1-null mutation leads to postnatal lethality due to constitutive Nrf2 activation. Nat Genet. 2003;35(3):238-245

30. Barretina J, et al. Subtype-specific genomic alterations define new targets for soft-tissue sarcoma therapy. Nat Genet. 2010;42(8):715-721.

31. Yagishita $\mathrm{Y}$, et al. Nrf2 protects pancreatic $\beta$-cells from oxidative and nitrosative stress in diabetic model mice. Diabetes. 2014;63(2):605-618

32. Yagishita Y, et al. Nrf2 improves leptin and insulin resistance provoked by hypothalamic oxidative stress. Cell Rep. 2017;18(8):2030-2044

33. Ji H, Weatherall P, Adams-Huet B, Garg A. Increased skeletal muscle volume in women with familial partial lipodystrophy, Dunnigan variety. J Clin Endocrinol Metab. 2013;98(8):E1410-E1413.

34. Cortés VA, et al. Molecular mechanisms of hepatic steatosis and insulin resistance in the AGPAT2-deficient mouse model of congenital generalized lipodystrophy. Cell Metab. 2009;9(2):165-176.

35. Sutinen J, Yki-Järvinen H. Increased resting energy expenditure, fat oxidation, and food intake in patients with highly active antiretroviral therapy-associated lipodystrophy. Am J Physiol Endocrinol Metab. 2007;292(3):E687-E692.

36. Valenti $\mathrm{L}$, et al. Hepatic notch signaling correlates with insulin resistance and nonalcoholic fatty liver disease. Diabetes. 2013;62(12):4052-4062

37. Koutnikova H, et al. Compensation by the muscle limits the metabolic consequences of lipodystrophy in PPAR gamma hypomorphic mice. Proc Natl Acad Sci USA. 2003;100(24):14457-14462. 
38. Meakin PJ, et al. Susceptibility of Nrf2-null mice to steatohepatitis and cirrhosis upon consumption of a high-fat diet is associated with oxidative stress, perturbation of the unfolded protein response, and disturbance in the expression of metabolic enzymes but not with insulin resistance. Mol Cell Biol. 2014;34(17):3305-3320.

39. Fisher FM, et al. Obesity is a fibroblast growth factor 21 (FGF21)-resistant state. Diabetes. 2010;59(11):2781-2789.

40. Domingo P, et al. Serum FGF21 levels are elevated in association with lipodystrophy, insulin resistance and biomarkers of liver injury in HIV-1-infected patients. AIDS. 2010;24(17):2629-2637.

41. Miehle K, et al. Serum concentrations of fibroblast growth factor 21 are elevated in patients with congenital or acquired lipodystrophy. Cytokine. 2016;83:239-244.

42. Kotronen A, et al. Hepatic stearoyl-CoA desaturase (SCD)-1 activity and diacylglycerol but not ceramide concentrations are increased in the nonalcoholic human fatty liver. Diabetes. 2009;58(1):203-208.

43. Duggan C, et al. Associations of insulin resistance and adiponectin with mortality in women with breast cancer. J Clin Oncol. 2011;29(1):32-39.

44. Nunez NP, et al. Accelerated tumor formation in a fatless mouse with type 2 diabetes and inflammation. Cancer Res. 2006;66(10):5469-5476.

45. Francis $P$, et al. Diagnostic and prognostic gene expression signatures in 177 soft tissue sarcomas: hypoxia-induced transcription profile signifies metastatic potential. BMC Genomics. 2007;8:73.

46. Bi P, et al. Notch activation drives adipocyte dedifferentiation and tumorigenic transformation in mice. J Exp Med 2016;213(10):2019-2037.

47. $\mathrm{Mu} \mathrm{X}$, et al. Notch signaling mediates skeletal muscle atrophy in cancer cachexia caused by osteosarcoma. Sarcoma. 2016;2016:3758162.

48. Hiramoto K, et al. Myeloid lineage-specific deletion of antioxidant system enhances tumor metastasis. Cancer Prev Res (Phila). 2014;7(8):835-844.

49. Itoh K, et al. An Nrf2/small Maf heterodimer mediates the induction of phase II detoxifying enzyme genes through antioxidant response elements. Biochem Biophys Res Commun. 1997;236(2):313-322.

50. Han H, et al. Inducible gene knockout of transcription factor recombination signal binding protein-J reveals its essential role in T versus B lineage decision. Int Immunol. 2002;14(6):637-645.

51. Wakabayashi N, et al. Notch-Nrf2 axis: regulation of Nrf2 gene expression and cytoprotection by notch signaling. Mol Cell Biol. 2014;34(4):653-663

52. Bligh EG, Dyer WJ. A rapid method of total lipid extraction and purification. Can J Biochem Physiol. 1959;37(8):911-917.

53. Fazzari M, et al. Nitro-fatty acid pharmacokinetics in the adipose tissue compartment. J Lipid Res. 2017;58(2):375-385.

54. Li X, Franke AA. Improved LC-MS method for the determination of fatty acids in red blood cells by LC-orbitrap MS. Anal Chem. 2011;83(8):3192-3198.

55. Pfaffl MW. A new mathematical model for relative quantification in real-time RT-PCR. Nucleic Acids Res. 2001;29(9):e45.

56. Andersen CL, Jensen JL, Ørntoft TF. Normalization of real-time quantitative reverse transcription-PCR data: a model-based variance estimation approach to identify genes suited for normalization, applied to bladder and colon cancer data sets. Cancer Res. 2004;64(15):5245-5250.

57. Wang X, Spandidos A, Wang H, Seed B. PrimerBank: a PCR primer database for quantitative gene expression analysis, 2012 update. Nucleic Acids Res. 2012;40(Database issue):D1144-D1149.

58. Li H, Durbin R. Fast and accurate short read alignment with Burrows-Wheeler transform. Bioinformatics. 2009;25(14):1754-1760

59. Li H, et al. The Sequence Alignment/Map format and SAMtools. Bioinformatics. 2009;25(16):2078-2079.

60. Koboldt DC, et al. VarScan 2: somatic mutation and copy number alteration discovery in cancer by exome sequencing. Genome Res. 2012;22(3):568-576.

61. Mayakonda A, Koeffler HP. Maftools: Efficient analysis, visualization and summarization of MAF files from large-scale cohort based cancer studies [published online ahead of print May 11, 2016]. bioRxiv. https://doi.org/10.1101/052662

62. Danecek P, et al. The variant call format and VCFtools. Bioinformatics. 2011;27(15):2156-2158.

63. Purcell S, et al. PLINK: a tool set for whole-genome association and population-based linkage analyses. Am J Hum Genet. 2007;81(3):559-575

64. Wolfe D, Dudek S, Ritchie MD, Pendergrass SA. Visualizing genomic information across chromosomes with PhenoGram BioData Min. 2013;6(1):18. 